RU01-12-B

hep-th/0108256

\title{
THE GINZBURG-LANDAU FREE ENERGY FUNCTIONAL OF COLOR SUPERCONDUCTIVITY AT WEAK COUPLING
}

\author{
Ioannis Giannakis ${ }^{a}$ and Hai-cang $\operatorname{Ren}^{a} \dagger$ \\ (a) Physics Department, The Rockefeller University \\ 1230 York Avenue, New York, NY 10021-6399
}

\begin{abstract}
We derive the Ginzburg-Landau free energy functional of color superconductivity in terms of the thermal diagrams of QCD in its perturbative region. The zero mode of the quadratic term coefficient yields the same transition temperature, including the pre-exponential factor, as the one obtained previously from the Fredholm determinant of the two quark scattering amplitude. All coefficients of the free energy can be made identical to those of a BCS model by setting the Fermi velocity of the latter equal to the speed of light. We also calculate the induced symmetric color condensate near $T_{c}$ and find that it scales as the cubic power of the dominant antisymmetric color component. We show that in the presence of an inhomogeneity and a nonzero gauge potential, while the color-flavor locked condensate dominates in the bulk, the unlocked condensate, the octet, emerges as a result of a simultaneous color-flavor rotation in the core region of a vortex filament or at the junction of super and normal phases.
\end{abstract}

$\dagger$ e-mail: giannak@theory.rockefeller.edu,ren@theory.rockefeller.edu, 
Ginzburg-Landau Free Energy Functional ...

\section{Introduction.}

The properties of hadronic matter under unusual conditions, high temperature or high baryon density, have been the subject of intense theoretical investigation, especially during the last few years. Relativistic heavy-ion collision experiments probe the quarkgluon plasma both at nonzero temperature and nonzero chemical potential. Quark matter at high baryon density and low temperature is expected to exist inside neutron stars. The exploration of these exotic states of matter will hopefully shed light on many nonperturbative aspects of quantum chromodynamics.

Although great progress has been achieved in our understanding of QCD along the temperature axis in the two-dimensional phase diagram of QCD with respect to temperature and chemical potential, based primarily in our ability to perform simulations on the lattice, the problem of low temperature, high density hadronic matter is less well understood mainly because the fermion sign problem at nonzero chemical potential makes numerical simulations practically impossible at high baryon density. Thus, many issues of the physics near the chemical potential axis can only be addressed analytically.

At ultra high chemical potential, $\mu>>\Lambda_{\mathrm{QCD}}$, the interaction between two quarks near their Fermi surface is dominated by one gluon exchange because of the asymptotic freedom. Such an interaction can be decomposed into a color symmetric channel and a color antisymmetric channel. For a pair of quarks within the color antisymmetric channel propagating in opposite directions, both color-electric and color-magnetic parts of the interaction are attractive. Therefore the Fermi surface is unstable against the formation of Cooper pairs and the quark matter becomes superconducting below a certain temperature [1]. Such a color superconducting state is expected to survive at medium high chemical potential, $\mu>\Lambda_{\mathrm{QCD}}(\mu=400 \sim 500 \mathrm{MeV})$, which corresponds to the baryon density achieved in RHIC and in the core of a typical neutron star. The instanton interaction may also contribute to the condensation at medium high chemical potential [2].

Theoretically, there are two approaches which explore the physics of the color superconductivity, one that utilizes an effective action involving only quark degrees of freedom while the other uses the fundamental QCD action. Using the first approach, important progress has been made by Alford, Rajagopal and Wilczek, who recognized that the colorflavor locked condensate represents the minimum of the bulk free energy and thus characterizes the super phase [3]. As this condensate possesses all the symmetries of low energy hadronic matter, the cross-over from one phase to the other is likely to be continuous [4]. The second approach [5],[6],[7], [8],[9], [10],[11], [12] yielded as a main result an asymptotic formula of the superconducting energy scale in the weak coupling limit. For example, the transition temperature with $N_{c}$ colors and $N_{f}$ flavors is given by

$$
k_{B} T_{C}=512 \pi^{3} e^{\gamma}\left(\frac{2}{N_{f}}\right)^{\frac{5}{2}} \frac{\mu}{g^{5}} e^{-\sqrt{\frac{6 N_{c}}{N_{c}+1}} \frac{\pi^{2}}{g}-\frac{\pi^{2}+4}{16}\left(N_{c}-1\right)}
$$


with $g$ being the QCD running coupling constant at $\mu$ and $\gamma=0.5772 .$. the Euler constant. The non-BCS behavior of the exponent stems from the long range propagation of the magnetic gluon being exchanged and the $O(1)$ term of the exponent is due to the suppression of the quasi-particle weight at the Fermi level, another consequence of the long range magnetic interaction. Other contributions which are subleading in $g$ [11], including the scheme dependence of the ultraviolet renormalization do not appear in (1.1). Remarkably, the formula (1.1), even when is extrapolated to the medium high chemical potential, gives rise to a very small ratio $k_{B} T_{C} / \mu$.

In this paper, we shall derive the Ginzburg-Landau free energy functional of color superconductivity at ultra high chemical potential. The conventional method of derivation was developed by Gorkov [13], who generalized the superconducting gap equation to a set of equations for ordinary and anomalous Green functions, the latter being appropriate to an inhomogeneous system. Although this formalism is easy to implement in the case of a fourfermion interaction it becomes rather cumbersome when one uses the fundamental QCD action with an energy dependent gap. Furthermore the systematics is not transparent. Instead, we shall employ the formulation developed by Jona-Lasinio [14], in which the Ginzburg-Landau free energy is identified as the generating functional of proper vertices. Each coefficient of the Ginzburg-Landau free energy is associated with a set of thermal diagrams in the normal phase. As we shall see the transition temperature corresponds to the zero mode of the inverse di-quark propagator and this criterion is rigorously equivalent to the one used in [10],[11], in which the transition temperature was identified with the zero of the Fredholm determinant of the di-quark scattering kernel. For a homogeneous system, the derived Ginzburg-Landau free energy agrees with the recent result of Iida and Baym [15] to the leading order in $g$ and in the absence of the color symmetric component of the condensate. The color-flavor locked condensate remains the energetically favored minimum in the bulk near $T_{C}$. Including the inhomogeneity, the Ginzburg-Landau free energy for QCD agrees exactly with that of a BCS superconductor upon setting the Fermi velocity of the latter to the speed of light, despite the non-BCS scaling of the transition temperature (1.1).

Although the one gluon exchange is repulsive in the color symmetric channel, a small component of the condensate in this channel was found to be induced at $T=0[3],[16]$, [17]. We shall analyze this mechanism as $T$ approaches $T_{C}$ from below. We find that the magnitude of the induced color symmetric condensate scales like the cube of that of the color antisymmetric condensate. The contribution to the free energy at equilibrium from the former is suppressed relative to that from the latter by a factor of the order of $\frac{\left(T_{C}-T\right)}{T_{C}}$, which is larger than the one that was speculated in [18].

As an important application of the Ginzburg-Landau theory, we shall also examine the coupling between the color-flavor locked condensate and the unlocked one through a gauge potential for an inhomogeneous system. We find the inevitable appearance of the unlocked condensate in the core region of a vortex filament or at the border of the super phase while the locked condensate remains dominant in the bulk. 
This paper is organized as follows: In section 2 we review the Jona-Lasinio method which we shall employ in this paper while in section 3 we discuss different characheristics of the order parameter of color superconductivity, which corresponds to a non-zero vacuum expectation value of a quark bilinear. Next in section 4 we calculate the Ginzburg-Landau free energy functional to the quadratic order in the order parameter and in section 5 we extend the calculation to the quartic order. Finally in section 6 we introduce a non-zero total momentum to the diquark condensate and the corresponding gauge coupling and examine the application to the realistic case with three colors and three flavors.

\section{General Formulation.}

In this section, we shall review the Jona-Lasinio method [14], which we shall employ in the microscopic derivation of the Ginzburg-Landau free energy functional of color superconductivity through out this paper.

Let $\mathcal{L}_{E}$ be the Lagrangian density which describes a field theoretic system in Euclidean space. The free energy of the system at temperature $T, F(T)$ can be expressed in terms of a functional integral

$$
e^{-\beta F(T)}=C \int \prod_{a} d \phi_{a} e^{-S_{E}(\phi)},
$$

where $\beta=1 / k_{B} T, S_{E}(\phi)=\int_{0}^{\beta} d \tau \int d^{3} \vec{r} \mathcal{L}_{E}(\phi)$ and $\phi_{a}$ stands for the collection of the field variables of the system with the subscript $a$ denoting its spacetime and internal coordinates. Periodic boundary conditions (anti-periodic) in Euclidean time $\tau$ are imposed on the bosonic fields (fermionic).

The order parameter of the system corresponds to the nonzero expectation value of an operator, $\Phi_{a}$, which may be one of the elementary fields of the theory or a composite operator and realize a nontrivial representation of the symmetry group of the system. To explore the stability of the system against the order parameter, we first trigger it with an external source, i.e.

$$
S_{E}(\phi) \rightarrow S_{E}^{J}(\phi)=S_{E}(\phi)+\sum_{a}\left(J_{a}^{*} \Phi_{a}+J_{a} \Phi_{a}^{*}\right)
$$

The corresponding shift of the free energy, $W(J)$, is given by

$$
e^{-\beta W(J)}=\frac{\int \prod_{a} d \phi_{a} e^{-S_{E}^{J}(\phi)}}{\int \prod_{a} d \phi_{a} e^{-S_{E}(\phi)}},
$$


and can be expanded in powers of $J$ and $J^{*}$, i.e.

$$
W(J)=-\frac{1}{\beta} \sum_{a b} \mathcal{D}_{a b} J_{a}^{*} J_{b}+\frac{1}{2 \beta} \sum_{a b c d} G_{a b c d} J_{a}^{*} J_{b}^{*} J_{c} J_{d}+O\left(|J|^{6}\right),
$$

where each coefficient corresponds to a set of connected diagrams that contains a fixed number of $J$ 's. In particular, $\mathcal{D}_{a b}$ corresponds to the propagator of $\Phi_{a}$. The order parameter triggered by $J$ reads

$$
B_{a} \equiv<\Phi_{a}>=\beta \frac{\delta W}{\delta J_{a}^{*}}=-\sum_{b} \mathcal{D}_{a b} J_{b}+\sum_{b c d} G_{a b c d} J_{b}^{*} J_{c} J_{d}+O\left(|J|^{5}\right)
$$

After performing a Legendre trasformation,

$$
\Gamma(B)=W(J)-\sum_{a}\left(J_{a}^{*} B_{a}+J_{a} B_{a}^{*}\right)
$$

we find that

$$
\frac{\delta \Gamma}{\delta B_{a}^{*}}=-J_{a}
$$

Inverting the series (2.5), we obtain that

$$
\Gamma(B)=\sum_{a b} \mathcal{M}_{a b} B_{a}^{*} B_{b}+\frac{1}{2} \sum_{a b c d} \mathcal{G}_{a b c d} B_{a}^{*} B_{b}^{*} B_{c} B_{d}+O\left(|B|^{6}\right)
$$

with

$$
\mathcal{M}_{a b}=\left(\mathcal{D}^{-1}\right)_{a b}
$$

and

$$
\mathcal{G}_{a b c d}=\sum_{a^{\prime} b^{\prime} c^{\prime} d^{\prime}} \mathcal{M}_{a a^{\prime}}^{*} \mathcal{M}_{b b^{\prime}}^{*} G_{a^{\prime} b^{\prime} c^{\prime} d^{\prime}} \mathcal{M}_{c c^{\prime}} \mathcal{M}_{d d^{\prime}}
$$

The functional $\Gamma(B)$ is nothing but the Ginzburg-Landau free energy functional of the order parameter. When the external source is removed, the minimization of $\Gamma$ at a nonzero $B$ signifies the condensate phase. For a second order phase transition, the sign of the quadratic term plays a decisive role.

Since the quartic term is positive and we are interested in the physics near $T_{c}$ we shall subsequently neglect higher terms. Few remarks are in order:

1. If the operator $\Phi_{a}$ is an elementary field or a quadratic function of the elementary fields, the manipulation leading from (2.2) to (2.8) can be carried out for a free field system with all the coefficients of (2.8) known explicitly. In this case $\Gamma$ is always positive for a nonzero $B$ and the only minimum of $\Gamma$ occurs at $B=0$. This is also the case with a repulsive interaction. 
2. The presence of an attractive interaction makes a difference. Let us diagonalize the matrix $\mathcal{M}$ and expand the order parameter $B$ in terms of the eigenmodes of $\mathcal{M}$. As we shall see below, in the presence of a Fermi sea, no matter how weak is the attraction, there is always a mode, whose corresponding eigenvalue becomes negative below a sufficiently low temperature $T_{C}$. We shall refer to this mode as the pairing mode in contrast to the others (whose eigenvalues are positive) which we shall refer to as non-pairing modes. Technically, we may switch off the interaction when dealing with the non-pairing modes in the leading order weak coupling approximation since the interaction is merely perturbative to them. The same approximation applies to the quartic term of (2.8), since the pairing singularity of $G_{a b c d}$ is cancelled by the zero of $\mathcal{M}$ 's upon amputation.

3. It is not always true that the order parameter which minimizes $\Gamma$ consists of only the pairing mode. In the presence of a term that is cubic in the pairing mode and linear in a nonpairing mode, an expectation value of the nonpairing mode will be induced upon minimization, the magnitude of which will be proportional to the cubic power of that of the pairing mode. As the critical temperature $T_{C}$ is approached from below, the pairing mode condensate scales as $\left(T_{C}-T\right)^{\frac{1}{2}}$ and the induced nonpairing mode scales as $\left(T_{C}-T\right)^{\frac{3}{2}}$. As we shall see below, this is the case with the sextet condensate in the color-flavor locked phase of three colors and three flavors.

4. As is the case for all mean field theories in three dimensions, the weak coupling calculation can not be extended to the temperature arbitrarily close to the critical temperature $T_{C}$, since the perturbative expansion in the coupling constant is not uniform in $T-T_{C}$. The size of the region where our approximation fails, the critical region, can be estimated according to the Ginzburg criterion. Because of the agreement of our derived Ginzburg-Landau free energy with that of a BCS model, we conclude that the width of the critical window is given by

$$
\frac{\left|T-T_{C}\right|}{T_{C}} \sim\left(\frac{k_{B} T_{C}}{\mu}\right)^{4}
$$

which is exceedingly narrow. Technically, the critical point prevents us from continuing $\Gamma(B)$ obtained in the normal phase to the super phase at $J=0$. A nonzero triggering source $J$ is required for a smooth cross over to the super phase. To be more specific, the normal phase $\Gamma$ functional can be carried over to the super phase following the path $A B C D$ in Fig.1.

In the appendix A, we shall utilize the Jona-Lasinio formulation in order to derive the Ginzburg-Landau free energy functional for a BCS model as an illurstration and as comparison to the analogous expression for color superconductivity. 
Ginzburg-Landau Free Energy Functional ...

\section{The Order Parameters of QCD at High Baryon Density.}

Consider now an $S U\left(N_{c}\right)$ color gauge theory coupled to $N_{f}$ flavors of massless quarks at temperature $T$ and chemical potential $\mu$. The Eucliden action of the system reads

$$
S_{E}=\int_{0}^{\beta} d \tau \int d^{3} \vec{r}\left[\frac{1}{4} F_{\mu \nu}^{l} F_{\mu \nu}^{l}-\bar{\psi} \gamma_{\mu}\left(\frac{\partial}{\partial x_{\mu}}-i g A_{\mu}\right) \psi+\mu \psi^{\dagger} \psi+\cdots\right],
$$

where $F_{\mu \nu}^{l}=\partial_{\mu} A_{\nu}^{l}-\partial_{\nu} A_{\mu}^{l}+g f^{l a b} A_{\mu}^{a} A_{\nu}^{b}, A_{\mu}=A_{\mu}^{l} T^{l}$ with $T^{l}$ being the $S U\left(N_{c}\right)$ generators in the fundamental representation and the dots represent gauge fixing and ghost terms. Since the action (3.1) is diagonal with respect to both flavor and chirality, the corresponding indices have been dropped in (3.1). Our gamma matrices are all hermitian with $\gamma_{5}=$ $\gamma_{1} \gamma_{2} \gamma_{3} \gamma_{4}$

The fermion field can be decomposed into eigenfunctions of $\gamma_{5}$, which in turn can be separated into the positive energy and negative energy parts

$$
\psi_{f}^{c}(\vec{r}, \tau)=\psi_{f L+}^{c}(\vec{r}, \tau)+\psi_{f L-}^{c}(\vec{r}, \tau)+\psi_{f R+}^{c}(\vec{r}, \tau)+\psi_{f R-}^{c}(\vec{r}, \tau),
$$

where we have restored the color-flavor indices for clarity. The Fourier expansion of $\psi_{f L(R) \pm}^{c}(\vec{r}, \tau)$ is given by

$$
\begin{aligned}
& \psi_{f L+}^{c}(\vec{r}, \tau)=\frac{1}{\sqrt{\beta \Omega}} \sum_{P} e^{i \vec{p} \cdot \vec{r}-i \nu_{n} \tau} a_{f, P, L}^{c} u_{L}(\vec{p}) \\
& \psi_{f L_{-}}^{c}(\vec{r}, \tau)=\frac{1}{\sqrt{\beta \Omega}} \sum_{P} e^{i \vec{p} \cdot \vec{r}-i \nu_{n} \tau} b_{f, P, L}^{c} v_{L}(-\vec{p}) \\
& \psi_{f R+}^{c}(\vec{r}, \tau)=\frac{1}{\sqrt{\beta \Omega}} \sum_{P} e^{i \vec{p} \cdot \vec{r}-i \nu_{n} \tau} a_{f, P, R}^{c} u_{R}(\vec{p}) \\
& \psi_{f L-}^{c}(\vec{r}, \tau)=\frac{1}{\sqrt{\beta \Omega}} \sum_{P} e^{i \vec{p} \cdot \vec{r}-i \nu_{n} \tau} b_{f, P, R}^{c} v_{R}(-\vec{p})
\end{aligned}
$$

and parallel expressions in terms of $\bar{a}_{f, P, L}^{c}, \bar{b}_{f, P, L}^{c}, \bar{a}_{f, P, R}^{c}$ and $\bar{b}_{f, P, R}^{c}$ exist for $\bar{\psi}_{f L(R) \pm}^{c}(\vec{r}, \tau)$, where $P=\left(\vec{p},-\nu_{n}\right)$ denotes an Euclidean four momentum with $\nu_{n}=2 n \pi k_{B} T(n=$ $\left.\pm \frac{1}{2}, \pm \frac{3}{2}, \ldots\right)$ the Matsubara energy. The four component spinors $u_{L(R)}(\vec{p})$ satisfy the Dirac equation $\left(\gamma_{4} p-i \vec{\gamma} \cdot \vec{p}\right) u_{L(R)}(\vec{p})=0$ and are normalized according to $u_{L(R)}^{\dagger}(\vec{p}) u_{L(R)}(\vec{p})=1$. Furthermore, we have $\gamma_{5} u_{L}(\vec{p})=u_{L}(\vec{p})$ and $\gamma_{5} u_{R}(\vec{p})=-u_{R}(\vec{p})$. Similarly $v_{L(R)}(\vec{p})$ obeys the Dirac equation and the chirality conditions. The symbol $\Omega$ stands for the volume of the system and as $\Omega \mapsto \infty$

$$
\frac{1}{\beta \Omega} \sum_{P} \mapsto \frac{1}{\beta} \sum_{n} \int \frac{d^{3} \vec{p}}{(2 \pi)^{3}}
$$


To trigger the long range order, we add to (3.1) a source term,

$$
\begin{aligned}
\Delta S & =\frac{1}{2} \sum_{h=L, R ; s= \pm} \int_{0}^{\beta} d \tau_{1} \int d^{3} \vec{r}_{1} \int_{0}^{\beta} d \tau_{2} \int d^{3} \vec{r}_{2}\left[\overline{\mathcal{J}}_{f_{1} f_{2}, h, s}^{c_{1} c_{2}}\left(\vec{r}_{1}, \tau_{1} ; \vec{r}_{2}, \tau_{2}\right)\right. \\
& \left.\tilde{\psi}_{f_{1}, h, s}^{c_{1}}\left(\vec{r}_{1}, \tau_{1}\right) \sigma_{2} \psi_{f_{2}, h, s}^{c_{2}}\left(\vec{r}_{2}, \tau_{2}\right)+\mathcal{J}_{f_{1} f_{2}, h, s}^{c_{1} c_{2}}\left(\vec{r}_{1}, \tau_{1} ; \vec{r}_{2}, \tau_{2}\right) \tilde{\bar{\psi}}_{f_{2}, h, s}^{c_{2}}\left(\vec{r}_{2}, \tau_{2}\right) \sigma_{2} \bar{\psi}_{f_{1}, h, s}^{c_{1}}\left(\vec{r}_{1}, \tau_{1}\right)\right]
\end{aligned}
$$

with

$$
\overline{\mathcal{J}}_{f_{1} f_{2}, h, s}^{c_{1} c_{2}}\left(\vec{r}_{1},-\tau_{1} ; \vec{r}_{2},-\tau_{2}\right)=\mathcal{J}_{f_{1} f_{2}, h, s}^{* c_{1} c_{2}}\left(\vec{r}_{1}, \tau_{1} ; \vec{r}_{2}, \tau_{2}\right)
$$

and

$$
\mathcal{J}_{f_{1} f_{2}, h, s}^{c_{1} c_{2}}\left(\vec{r}_{1}, \tau_{1} ; \vec{r}_{2}, \tau_{2}\right)=\mathcal{J}_{f_{2} f_{1}, h, s}^{c_{2} c_{1}}\left(\vec{r}_{2}, \tau_{2} ; \vec{r}_{1}, \tau_{1}\right) .
$$

The condition (3.6) is the Wick rotation of the complex conjugation with a real time and the symmetry property (3.7) is evident from the anticommutation relation of the $\psi$ 's and the antisymmetry of $\sigma_{2}$. Both of them are shared by the induced order parameter

$$
\mathcal{B}_{f_{1} f_{2}, h, s}^{c_{1} c_{2}}\left(\vec{r}_{1}, \tau_{1} ; \vec{r}_{2}, \tau_{2}\right) \equiv<\tilde{\psi}_{f_{1}, h, s}^{c_{1}}\left(\vec{r}_{1}, \tau_{1}\right) \sigma_{2} \psi_{f_{2}, h, s}^{c_{2}}\left(\vec{r}_{2}, \tau_{2}\right)>
$$

To trigger a homogeneous condensate, the source should depend only on the relative Euclidean coordinates, $\left(\vec{r}_{1}-\vec{r}_{2}, \tau_{1}-\tau_{2}\right)$ and its Fourier expansion reads

$$
\mathcal{J}_{f_{1} f_{2}, h, s}^{c_{1} c_{2}}\left(\vec{r}_{1}, \tau_{1} ; \vec{r}_{2}, \tau_{2}\right)=\frac{1}{\beta \Omega} \sum_{P} e^{i \vec{p} \cdot\left(\vec{r}_{1}-\vec{r}_{2}\right)-i \nu_{n}\left(\tau_{1}-\tau_{2}\right)} J_{f_{1} f_{2}, h, s}^{c_{1} c_{2}}(P)
$$

with $J_{f_{1} f_{2}, h, s}^{c_{1} c_{2}}(P)=J_{f_{2} f_{1}, h, s}^{c_{2} c_{1}}(-P)$. Correspondingly,

$$
\mathcal{B}_{f_{1} f_{2}, h, s}^{c_{1} c_{2}}\left(\vec{r}_{1}, \tau_{1} ; \vec{r}_{2}, \tau_{2}\right)=\frac{1}{\beta \Omega} \sum_{P} e^{i \vec{p} \cdot\left(\vec{r}_{1}-\vec{r}_{2}\right)-i \nu_{n}\left(\tau_{1}-\tau_{2}\right)} B_{f_{1} f_{2}, h, s}^{c_{1} c_{2}}(P)
$$

with $B_{f_{1} f_{2}, h, s}^{c_{1} c_{2}}(P)=B_{f_{2} f_{1}, h, s}^{c_{2} c_{1}}(-P)$. The triggering part of the action (3.5) becomes

$$
\Delta S=\sum_{P}^{\prime}\left[\bar{J}_{f_{1} f_{2}, h, s}^{c_{1} c_{2}}(P) a_{f_{2},-P}^{c_{2}} a_{f_{1}, P}^{c_{1}}+J_{f_{1} f_{2}, h, s}^{c_{1} c_{2}}(P) \bar{a}_{f_{1}, P}^{c_{1}} \bar{a}_{f_{2},-P}^{c_{2}}\right]
$$

with the sum over $P$ extending only to the half $\vec{p}$-space and $\bar{J}=J^{\star}$.

As the condensate consisting of quarks of left helicity contributes identically with the condensate of quarks of right helicity the total Ginzburg-Landau free energy functioncal of the system can be written

$$
\Gamma_{\text {total }}\left(\mathcal{B}_{L}, \mathcal{B}_{R}\right)=\Gamma\left(\mathcal{B}_{L}\right)+\Gamma\left(\mathcal{B}_{R}\right)+\cdots
$$

where the $\cdots$ indicate terms which include both $\mathcal{B}_{L}$ and $\mathcal{B}_{R}$ and arise due to instanton effects [19], [20]. Such terms are exponentially small in comparison with the first two terms 
of (3.12) but nevertheless contribute to fix the relative phase between $\mathcal{B}_{L}$ and $\mathcal{B}_{R}$. Since the even parity is favored for the ground state, we expect that

$$
\mathcal{B}_{f_{1} f_{2}, L, s}^{c_{1} c_{2}}\left(\vec{r}_{1}, \tau_{1} ; \vec{r}_{2}, \tau_{2}\right)=\mathcal{B}_{f_{1} f_{2}, R, s}^{c_{1} c_{2}}\left(-\vec{r}_{1}, \tau_{1} ;-\vec{r}_{2}, \tau_{2}\right)=\mathcal{B}_{f_{1} f_{2}, s}^{c_{1} c_{2}}\left(\vec{r}_{1}, \tau_{1} ; \vec{r}_{2}, \tau_{2}\right)
$$

and (3.12) becomes

$$
\Gamma_{\text {total }}\left(\mathcal{B}_{L}, \mathcal{B}_{R}\right) \cong 2 \Gamma(\mathcal{B})
$$

where we have omitted the extra terms in $(3.12) . \Gamma(\mathcal{B})$ is the Ginzburg-Landau free energy functional with a single helicity. It's quartic expansion

$$
\Gamma(\mathcal{B})=\Gamma_{2}(\mathcal{B})+\Gamma_{4}(\mathcal{B})
$$

will be evaluated in the subsequent sections with the helicity subscripts $\mathrm{L}, \mathrm{R}$ omitted for clarity of notation.

At high baryon density, the pairing correlation extends hardly to the states below the Dirac sea and we may set $\mathcal{J}_{-}=0$ with $\mathcal{J}_{+} \equiv \mathcal{J}$.

In this paper, we shall consider only the $s$-wave pairing for which both $J_{f_{1} f_{2}}^{c_{1} c_{2}}(P)$ and $B_{f_{1} f_{2}}^{c_{1} c_{2}}(P)$ are independent of the direction of $\vec{p}$. As we shall see in the next section, the attractive force around the Fermi surface picks the order parameter which is even with respect to the sign of $\nu_{n}$ for pairing. Therefore

$$
J_{f_{1} f_{2}}^{c_{1} c_{2}}(P)=J_{f_{1} f_{2}}^{c_{1} c_{2}}(-P)
$$

and

It follows from (3.7) that

$$
B_{f_{1} f_{2}}^{c_{1} c_{2}}(P)=B_{f_{1} f_{2}}^{c_{1} c_{2}}(-P)
$$

$$
J_{f_{1} f_{2}}^{c_{1} c_{2}}(P)=J_{f_{2} f_{1}}^{c_{2} c_{1}}(P)
$$

and

$$
B_{f_{1} f_{2}}^{c_{1} c_{2}}(P)=B_{f_{2} f_{1}}^{c_{2} c_{1}}(P) \text {. }
$$

In other words, the triggering source and the order parameter are symmetric with respect to simultaneous exchange of color and flavor indices. According to the antisymmetric and symmetric representations of the color group, we decompose further

$$
B_{f_{1} f_{2}}^{c_{1} c_{2}}(P)=\phi_{f_{1} f_{2}}^{c_{1} c_{2}}(P)+\chi_{f_{1} f_{2}}^{c_{1} c_{2}}(P)
$$

with

$$
\phi_{f_{1} f_{2}}^{c_{1} c_{2}}(P)=-\phi_{f_{1} f_{2}}^{c_{2} c_{1}}(P)=-\phi_{f_{2} f_{1}}^{c_{1} c_{2}}(P)=\phi_{f_{2} f_{1}}^{c_{2} c_{1}}(P)
$$

and

$$
\chi_{f_{1} f_{2}}^{c_{1} c_{2}}(P)=\chi_{f_{1} f_{2}}^{c_{2} c_{1}}(P)=\chi_{f_{2} f_{1}}^{c_{1} c_{2}}(P)=\chi_{f_{2} f_{1}}^{c_{2} c_{1}}(P)
$$

with $\phi_{f_{1} f_{2}}^{c_{1} c_{2}}(P)$ pertaining to the attractive channel and $\chi_{f_{1} f_{2}}^{c_{1} c_{2}}(P)$ to the repulsive channel. The color-flavor locked condensate for $N_{c}=N_{f}$ corresponds, up to color-flavor-baryon number rotations, to

$$
B_{f_{1} f_{2}}^{c_{1} c_{2}}(P)=B_{1}(P) \delta_{f_{1}}^{c_{1}} \delta_{f_{2}}^{c_{2}}+B_{2}(P) \delta_{f_{1}}^{c_{2}} \delta_{f_{2}}^{c_{1}}
$$

which gives rise to the minimum free energy of a homogeneous system below $T_{C}$. 
Ginzburg-Landau Free Energy Functional ...

\section{The Di-Quark Propagator of QCD at High Baryon Density.}

The coefficient of $\Gamma_{2}(\mathcal{B})$ of $(2.8)$ is the inverse of the di-quark propagator, which corresponds to the thermal diagrams in Fig.2, when it is sandwiched by two order parameters. For total zero momentum and total zero Matsubara energy, the propagator is specified by the relative momentum, relative energy and color-flavor indices at both terminals. In writing, we have

$$
\begin{array}{r}
<P^{\prime}, s_{1}^{\prime}, s_{2}^{\prime} ; c_{1}^{\prime}, c_{2}^{\prime} ; f_{1}^{\prime}, f_{2}^{\prime}|\mathcal{D}| \\
P, s_{1}, s_{2} ; c_{1}, c_{2} ; f_{1}, f_{2}>=\delta^{c_{1}^{\prime} c_{1}} \delta^{c_{2}^{\prime} c_{2}} \delta_{f_{1}^{\prime} f_{1}} \delta_{f_{2}^{\prime} f_{2}} \delta_{P^{\prime} P} D_{s_{1}^{\prime} s_{2}^{\prime}, s_{1} s_{2}}(P) \\
+\delta_{f_{1}^{\prime} f_{1}} \delta_{f_{2}^{\prime} f_{2}} \sum_{t_{1}^{\prime} t_{2}^{\prime}, t_{1} t_{2}} D_{s_{1}^{\prime} s_{2}^{\prime}, t_{1}^{\prime} t_{2}^{\prime}}\left(P^{\prime}\right) \Gamma_{t_{1}^{\prime} t_{2}^{\prime}, t_{1} t_{2}}^{c_{1}^{\prime} c_{2}^{\prime}, c_{1} c_{2}}\left(P^{\prime} \mid P\right) D_{t_{1} t_{2}, s_{1} s_{2}}(P),
\end{array}
$$

where $D$ represents the disconnected diagrams of the di-quark propagator, the first diagram of Fig. 2a, $\Gamma$ is the sum of the proper vertices of the scattering between two quarks with zero total momentum and energy, and $P=\left(\vec{p},-\nu_{n}\right)$ denotes an Euclidean four-momentum. The superscripts $c_{1}, c_{2}, c_{1}^{\prime}, c_{2}^{\prime}$ stand for color indices, the subscripts $f_{1}, f_{2}, f_{1}^{\prime}, f_{2}^{\prime}$ denote the flavor indices and the subscripts $s_{1}, s_{2}, s_{1}^{\prime}, s_{2}^{\prime}$ and $t_{1}, t_{2}, t_{1}^{\prime}, t_{2}^{\prime}$ label the states above or below the Dirac sea. In order to facilitate the partial wave analysis, we found it convenient to associate the Dirac spinors $u(\vec{p})$ and $v(\vec{p})$ to the vertex instead of the propagator. Thus the vertex in (4.1) is of the form

$$
\Gamma_{s_{1}^{\prime} s_{2}^{\prime}, s_{1} s_{2}}^{c_{1}^{\prime} c_{2}^{\prime}, c_{1} c_{2}}\left(P^{\prime} \mid P\right)=\bar{U}_{\gamma}\left(s_{1}^{\prime}, \vec{p}^{\prime}\right) \bar{U}_{\delta}\left(s_{2}^{\prime},-\vec{p}^{\prime}\right) \Gamma_{\gamma \delta \alpha \beta}^{c_{1}^{\prime} c_{2}^{\prime}, c_{1} c_{2}}\left(P^{\prime} \mid P\right) U_{\alpha}\left(s_{1}, \vec{p}\right) U_{\beta}\left(s_{2},-\vec{p}\right)
$$

with the vertex function $\Gamma_{\gamma \delta \alpha \beta}$ given by conventional Feynman rules and $U(s, \vec{p})=u(s \vec{p})$. The overall sign of $(4.1)$ is fixed such that $D_{++++}(P) \mapsto \frac{1}{\nu_{n}^{2}+(p-\mu)^{2}}$ as $g \rightarrow 0$.

The proper vertex function $\Gamma$ satisfies the Schwinger-Dyson equation:

$$
\begin{aligned}
\Gamma_{s_{1}^{\prime} s_{2}^{\prime}, s_{1} s_{2}}^{c_{1}^{\prime} c_{2}^{\prime}, c_{1} c_{2}}\left(P^{\prime} \mid P\right) & =\bar{\Gamma}_{s_{1}^{\prime} s_{2}^{\prime}, s_{1} s_{2}}^{c_{\prime}^{\prime} c^{\prime}, c_{1} c_{2}}\left(P^{\prime} \mid P\right) \\
& +\frac{1}{\beta \Omega} \sum_{P^{\prime \prime} ; t_{1}^{\prime}, t_{2}^{\prime}, t_{1}, t_{2} ; c_{1}^{\prime \prime}, c_{2}^{\prime \prime}} \bar{\Gamma}_{s_{1}^{\prime} s_{2}^{\prime}, t_{1}^{\prime} t_{2}^{\prime}}^{c_{1}^{\prime} c_{2}^{\prime \prime}, P_{1}^{\prime \prime}}\left(P^{\prime} \mid P^{\prime \prime}\right) D_{t_{1}^{\prime} t_{2}^{\prime} t_{1} t_{2}}\left(P^{\prime \prime}\right) \Gamma_{t_{1} t_{2}, s_{1} s_{2}}^{c_{1}^{\prime \prime} c_{2}^{\prime \prime}, c_{1} c_{2}}\left(P^{\prime \prime} \mid P\right)
\end{aligned}
$$

with $\bar{\Gamma}$ standing for two particle irreducible vertices. In terms of matrix notations, (4.1) and (4.3) take the form

$$
\mathcal{D}=D-D \Gamma D
$$

and

$$
\Gamma=\bar{\Gamma}+\bar{\Gamma} D \Gamma
$$


Combining (4.4) and (4.5), we find a simple relationship

$$
\mathcal{M}=\mathcal{D}^{-1}=D^{-1}+\bar{\Gamma}
$$

which generalizes the diagrammatics of a single particle propagator in terms of one particle irreducible self energy function. Upon decomposition of $\bar{\Gamma}$ into its symmetric (sextet color representation of $S U\left(N_{c}\right)$ ) and antisymmetric (anti-triplet color representation of $S U\left(N_{c}\right)$ ) components,

$$
\begin{aligned}
\bar{\Gamma}_{s_{1}^{\prime} s_{2}^{\prime}, s_{1} s_{2}}^{c_{1}^{\prime}, c_{1} c_{2}}\left(P^{\prime} \mid P\right) & =\frac{1}{2}\left(\delta^{c_{1}^{\prime} c_{1}} \delta^{c_{2}^{\prime} c_{2}}-\delta^{c_{1}^{\prime} c_{2}} \delta^{c_{2}^{\prime} c_{1}}\right) \bar{\Gamma}_{s_{1}^{\prime} s_{2}^{\prime}, s_{1} s_{2}}^{A}\left(P^{\prime} \mid P\right) \\
& +\frac{1}{2}\left(\delta^{c_{1}^{\prime} c_{1}} \delta^{c_{2}^{\prime} c_{2}}+\delta^{c_{1}^{\prime} c_{2}} \delta^{c_{2}^{\prime} c_{1}}\right) \bar{\Gamma}_{s_{1}^{\prime} s_{2}^{\prime}, s_{1} s_{2}}^{S}\left(P^{\prime} \mid P\right)
\end{aligned}
$$

we obtain

$$
\begin{aligned}
& <P^{\prime}, s_{1}^{\prime}, s_{2}^{\prime} ; c_{1}^{\prime}, c_{2}^{\prime} ; f_{1}^{\prime}, f_{2}^{\prime}|\mathcal{M}| P, s_{1}, s_{2} ; c_{1}, c_{2} ; f_{1}, f_{2}>=\frac{1}{2} \delta_{f_{1}^{\prime} f_{1}} \delta_{f_{2}^{\prime} f_{2}}\left[\left(\delta^{c_{1}^{\prime} c_{1}} \delta^{c_{2}^{\prime} c_{2}}-\delta^{c_{1}^{\prime} c_{2}} \delta^{c_{2}^{\prime} c_{1}}\right)\right. \\
& \left.<P^{\prime}, s_{1}^{\prime}, s_{2}^{\prime}\left|\mathcal{M}_{A}\right| P, s_{1}, s_{2}>+\left(\delta^{c_{1}^{\prime} c_{1}} \delta^{c_{2}^{\prime} c_{2}}+\delta^{c_{1}^{\prime} c_{2}} \delta^{c_{2}^{\prime} c_{1}}\right)<P^{\prime}, s_{1}^{\prime}, s_{2}^{\prime}\left|\mathcal{M}_{S}\right| P, s_{1}, s_{2}>\right]
\end{aligned}
$$

with

$$
<P^{\prime}, s_{1}^{\prime}, s_{2}^{\prime}\left|\mathcal{M}_{A(S)}\right| P, s_{1}, s_{2}>=\delta_{P^{\prime} P} D_{s_{1}^{\prime} s_{2}^{\prime}, s_{1} s_{2}}^{-1}(P)+\frac{1}{\beta \Omega} \bar{\Gamma}_{s_{1}^{\prime} s_{2}^{\prime}, s_{1} s_{2}}^{A(S)}\left(P^{\prime} \mid P\right) .
$$

To the order of the approximation made in this work, the states below the Dirac sea do not contribute. We shall consider only the channels with all states above the Dirac sea and have the subscripts $s$ 's and $t$ 's suppressed.

By calculating the matrix elements of $\mathcal{M}$ between the order parameter (3.20), we obtain formally the quadratic term of the Ginzburg-Landau free energy functional with a single helicity

$$
\Gamma_{2}(\mathcal{B})=\frac{1}{2 \beta} \sum_{P^{\prime}, P}\left[<P^{\prime}\left|\mathcal{M}_{A}\right| P>\operatorname{Tr} \phi^{\dagger}\left(P^{\prime}\right) \phi(P)+<P^{\prime}\left|\mathcal{M}_{S}\right| P>\operatorname{Tr} \chi^{\dagger}\left(P^{\prime}\right) \chi(P)\right]
$$

where we regard $\phi_{f_{1} f_{2}}^{c_{1} c_{2}}$ and $\chi_{f_{1} f_{2}}^{c_{1} c_{2}}$ matrix elements of $N_{c} N_{f} \times N_{c} N_{f}$ matrices $\phi$ and $\chi$. The symmetry factor $\frac{1}{2}$ stems from the fact that we are only considering the condensate that consists of quarks of left helicity. With the HDL resummation, the irreducible vertex for the two quark scattering above the Dirac sea reads

$$
\bar{\Gamma}_{A}\left(P^{\prime} \mid P\right) \simeq-\frac{g^{2}}{12 p p^{\prime}}\left(1+\frac{1}{N_{c}}\right) \ln \frac{1}{\left|\hat{\nu}_{n}-\hat{\nu}_{n^{\prime}}\right|}+\text { higher partial wave components }
$$

with

$$
\hat{\nu}=\frac{g^{5}}{256 \pi^{4}}\left(\frac{N_{f}}{2}\right)^{\frac{5}{2}} \frac{\nu}{\mu}
$$


and the function $D^{-1}$ for two quarks propagating above Dirac sea is given by

$$
D^{-1}(P)=\left[i \nu_{n}-p+\mu-\Sigma\left(i \nu_{n}, p\right)\right]\left[-i \nu_{n}-p+\mu-\Sigma\left(-i \nu_{n}, p\right)\right]
$$

with the single quark self-energy

$$
\Sigma\left(i \nu_{n}, p\right) \simeq-i \frac{g^{2}}{12 \pi^{2}} \frac{N_{c}^{2}-1}{2 N_{c}} \nu_{n} \ln \frac{4 q_{c}^{3}}{\pi m_{D}^{2}\left|\nu_{n}\right|},
$$

where $m_{D}=\frac{N_{f} g^{2} \mu^{2}}{2 \pi^{2}}$ denotes the Debye mass and $q_{c}$ is an infrared cutoff $\left(\left|\nu_{n}\right|<<q_{c}<<\mu\right)$. As it was shown in [11], the weak coupling approximation in (4.11) and (4.13) is sufficient to determine the leading order of the pre-exponential factor of the transition temperature (1.1) or the gap energy. Since the color-symmetric channel does not carry the pairing modes, we may set

$$
<P^{\prime}\left|\mathcal{M}_{S}\right| P>\simeq D_{0}^{-1}(P) \delta_{P^{\prime} P}
$$

with $D_{0}(P)=\frac{1}{\nu_{n}^{2}+(p-\mu)^{2}}$ the free di-quark thermal propagator.

The spectrum of the operator $\mathcal{M}_{A}$ is given by the eigenvalue problem,

$$
\frac{1}{\beta \Omega} \sum_{P^{\prime}}<P\left|\mathcal{M}_{A}\right| P^{\prime}>u\left(P^{\prime}\right)=E u(P)
$$

i.e.

$$
D^{-1}(P) u(P)+\frac{1}{\beta \Omega} \sum_{P^{\prime}} \Gamma_{A}\left(P \mid P^{\prime}\right) u\left(P^{\prime}\right)=E u(P)
$$

Introducing

$$
f(P) \equiv-\frac{1}{\beta \Omega} \sum_{P^{\prime}} \Gamma_{A}\left(P \mid P^{\prime}\right) u\left(P^{\prime}\right)
$$

we have

$$
u(P)=\frac{f(P)}{D^{-1}(P)-E}
$$

Substituting (4.18) back to (4.17), we find that the function $f(P)$ satisfies a homogeneous Fredholm equation

$$
f(P)=\frac{1}{\beta \Omega} \sum_{P^{\prime}} K_{E}\left(P \mid P^{\prime}\right) f\left(P^{\prime}\right)
$$

with the kernel given by

$$
K_{E}\left(P^{\prime} \mid P\right)=-\frac{\Gamma_{A}\left(P^{\prime} \mid P\right)}{D^{-1}(P)-E}
$$


In order to demonstrate the equivalence with the methodology used in [10],[11] to determine the transition temperature, we switch to the eigenvalue problem defined by the Fredholm equation

$$
h(P)=\frac{\lambda^{2}}{\beta \Omega} \sum_{P^{\prime}} K_{E}\left(P \mid P^{\prime}\right) h\left(P^{\prime}\right)
$$

with the eigenvalues $\lambda^{2}$ 's functions of the temperature, chemical potential and $E$. The eq. (4.19) implies that

$$
\lambda^{2}(T, \mu, E)=1
$$

which determines $E$ as function of $T$ and $\mu$. For the pairing mode, we expect that $E=0$ at $T=T_{C}$, i.e. $\lambda^{2}\left(T_{C}, \mu, 0\right)=1$, which is exactly the criterion for $T_{C}$ in [11]. As $T$ gets sufficiently close to $T_{C}$, we may write

$$
K_{E}\left(P \mid P^{\prime}\right)=K\left(P \mid P^{\prime}\right)+\delta K\left(P \mid P^{\prime}\right)
$$

with $K\left(P \mid P^{\prime}\right)=-\Gamma_{A}\left(P \mid P^{\prime}\right) D\left(P^{\prime}\right)$ and $\delta K\left(P \mid P^{\prime}\right)=-\Gamma_{A}\left(P \mid P^{\prime}\right) D^{2}\left(P^{\prime}\right) E$. Correspondingly,

$$
1=\lambda^{-2}(T, \mu, 0)+c(T, \mu) E,
$$

with the coefficient $c(T, \mu)$ being calculated perturbatively. The eigenvalues of the kernel at $E=0$ and the transition temperature have been explored in [10], [11], and the one that satisfies the condition $\lambda^{2}\left(T_{C}, \mu, 0\right)=1$ is given, in the vicinity of $T_{C}$, by

$$
\frac{1}{\lambda^{2}}=\frac{g^{2}}{6 \pi^{4}}\left(1+\frac{1}{N_{c}}\right) \ln ^{2} \frac{1}{\epsilon}\left[1+2 \frac{\gamma+\ln 2}{\ln \frac{1}{\epsilon}}-\frac{g^{2}}{48 \pi^{4} N_{c}}\left(N_{c}^{2}-1\right)\left(\pi^{2}+4\right) \ln \frac{1}{\epsilon}+O\left(g^{2}\right)\right]
$$

where

$$
\epsilon=\left(\frac{N_{f}}{2}\right)^{\frac{5}{2}} \frac{g^{5} k_{B} T}{256 \pi^{3} \mu}
$$

and $\ln \frac{1}{\epsilon} \sim \frac{1}{g}$, so that the 2 nd and 3rd term inside the bracket are of the same order, $O(g)$. For $\left|T-T_{C}\right|<<T_{C}$, we have

$$
\begin{aligned}
1-\frac{1}{\lambda^{2}(T, \mu, 0)} & \simeq \frac{g^{2}}{3 \pi^{4}}\left(1+\frac{1}{N_{c}}\right)\left[\ln \frac{1}{\epsilon_{C}}+\gamma+\ln 2-\frac{3}{16}(N-1)\left(\pi^{2}+4\right)\right] \frac{T-T_{C}}{T_{C}} \\
& \simeq \frac{g^{2}}{3 \pi^{4}}\left(1+\frac{1}{N_{c}}\right) \ln \frac{1}{\epsilon_{C}} \frac{T-T_{C}}{T_{C}},
\end{aligned}
$$

with $\epsilon_{c}=\left.\epsilon\right|_{T=T_{c}}$. The constant pertaining the logarithm in the braket of (4.27), which contributes to the pre-exponential factor of the transition temperature (1.1) is subleading in the coefficient of $T-T_{c}$ in comparison with $\ln \frac{1}{\epsilon_{C}}$ and is dropped in the last step.

For the rest of the calculation, we shall set $T=T_{C}$ and maintain only the leading order term in $g$. Under such an approximation, the eigenfunction in (4.21) at $E=0$ is given by [11]

$$
h(P)=\frac{4 \pi^{2}}{\sqrt{7 \zeta(3)}} \frac{k_{B} T_{C}}{p} \sin \left(\frac{\pi \ln \frac{1}{\hat{\nu}_{n}}}{2 \ln \frac{1}{\epsilon_{C}}}\right) \theta\left(\delta-\left|\nu_{n}\right|\right)
$$


with $\delta$ a cutoff energy such that $k_{B} T_{C}<<\delta<<\mu$, and the eigenfunction $u(P)$ in (4.18) that corresponds to the pairing mode reads

$$
u(P)=\frac{h(P)}{\nu_{n}^{2}+(p-\mu)^{2}} .
$$

The choice of the constant in front of the sine of (4.28) normalizes $u(P)$ according to

$$
\frac{1}{\beta_{C} \Omega} \sum_{P}|u(P)|^{2}=1 .
$$

By sandwiching (4.23) between $h(P)$ and its adjoint, $\bar{h}(P) \simeq \frac{h(n, \vec{p})}{\nu_{n}^{2}+(p-\mu)^{2}}$, and subsequently summing over $P$, we find that

$$
c\left(T_{C}, \mu\right)=\frac{7 \zeta(3)}{4 \pi^{2} k_{B}^{2} T_{C}^{2} \ln \frac{1}{\epsilon_{C}}} .
$$

It follows from (4.24), (4.27) and (4.31) that

$$
E \simeq \frac{8 \pi^{2}}{7 \zeta(3)} k_{B}^{2} T_{C}\left(T-T_{C}\right)
$$

Note that, the summations over $n$ in (4.30) and (4.31) are dominated by the terms with $|n|=O(1)$ and the energy dependence of $h(P)$ may be dropped to the leading order in $g$.

Upon the decomposition of $\phi$ into its pairing mode and non-pairing modes,

$$
\phi=\phi_{0}+\phi^{\prime}
$$

with

$$
\phi_{0}=\sqrt{6} \Psi u(P)
$$

and

$$
\frac{1}{\beta \Omega} \sum_{P} \phi_{0}^{\dagger}(P) \phi^{\prime}(P)=0
$$

the quadratic term of the Ginzburg-Landau functional (4.10) becomes

$\Gamma_{2}(\mathcal{B})=\Omega \frac{24 \pi^{2}}{7 \zeta(3)} k_{B}^{2} T_{C}\left(T-T_{C}\right) \operatorname{Tr} \Psi^{\dagger} \Psi+\frac{1}{2 \beta}\left\{\sum_{P}\left[\nu_{n}^{2}+(p-\mu)^{2}\right] \operatorname{Tr}\left[\phi^{\prime \dagger}(P) \phi^{\prime}(P)+\chi^{\prime}(P) \chi(P)\right]\right\}$

where $\Psi$ is a constant $N_{c} N_{f} \times N_{c} N_{f}$ matrix, the function $u(P)$ is given by (4.29) and we have made a zero-th order approximation (4.14), to the coefficients in front of the non-pairing modes. 


\section{The Quartic Terms.}

Because of the elimination of the pairing singularity in the diquark scattering channel embedded in the diagrams $\mathcal{G}$ upon amputation, we may proceed with $g=0$. The action becomes then quadratic and the path integral in the presence of the triggering term can be evaluated explicitly.

Ignoring the Yang-Mills part and the fermionic states below the Dirac sea, the action (3.1) becomes

$S_{E}(a, \bar{a})=\sum_{P}\left(-i \nu_{n}+p-\mu\right) \bar{a}_{f, P}^{c} a_{f, P}^{c}+\sum_{P}^{\prime}\left[J_{f_{1} f_{2}}^{c_{1} c_{2}}(P)^{*} a_{f_{2},-P}^{c_{2}} a_{f_{1}, P}^{c_{1}}+J_{f_{1} f_{2}}^{c_{1} c_{2}}(P) \bar{a}_{f_{1}, P}^{c_{1}} \bar{a}_{f_{2},-P}^{c_{2}}\right]$,

where the repeated color-flavor indices are summed and the second sum, $\sum_{P}^{\prime}$ extends only half $\vec{p}$-space. The triggering source for a given $P=\left(\vec{p},-\nu_{n}\right), J_{f_{1} f_{2}}^{c_{1} c_{2}}(P)$ is an element of a $N_{c} N_{f} \times N_{c} N_{f}$ matrix $J(P)$, and the free energy shift is given by

$$
\begin{aligned}
W(J) & =-k_{B} T_{C} \sum_{P}^{\prime} \ln \frac{\operatorname{det}\left(\begin{array}{cc}
-i \nu_{n}+p-\mu & J(P) \\
J^{\dagger}(P) & -i \nu_{n}-p+\mu
\end{array}\right)}{\operatorname{det}\left(\begin{array}{cc}
-i \nu_{n}+p-\mu & 0 \\
0 & -i \nu_{n}-p+\mu
\end{array}\right)} \\
& =-\frac{k_{B} T_{C}}{2} \sum_{P} \frac{\operatorname{Tr} J^{\dagger}(P) J(P)}{\nu_{n}^{2}+(p-\mu)^{2}}+\frac{k_{B} T_{C}}{4} \sum_{P} \frac{\operatorname{Tr}\left[J^{\dagger}(P) J(P)\right]^{2}}{\left[\nu_{n}^{2}+(p-\mu)^{2}\right]^{2}}+\ldots
\end{aligned}
$$

which corresponds to the sum of the ring diagrams in Fig. 3. Note the symmetry factor $\frac{1}{2}$ of each term because of the single helicity pairing. Through a Legendre transformation, we obtain the quartic term of the Ginzburg- Landau free energy functional with a single helicity in terms of the order parameters

$$
\Gamma_{4}(\mathcal{B})=\frac{k_{B} T_{C}}{4} \sum_{P}\left[\nu_{n}^{2}+(p-\mu)^{2}\right]^{2} \operatorname{Tr}\left[B^{\dagger}(P) B(P)\right]^{2},
$$

the coefficient of which is valid to the leading order of the coupling constant $g$. Upon the decomposition of the order parameter $B$ into its color antisymmetric component and color symmetric one, eq.(3.20) and further decomposition of the color antisymmetric component into its pairing mode and non-pairing modes, (4.33), we arrive at

$$
\begin{aligned}
\Gamma_{4}(\mathcal{B}) & =\frac{k_{B} T_{C}}{4} \sum_{P}\left[\nu_{n}^{2}+(p-\mu)^{2}\right]^{2} \operatorname{Tr}\left\{\left[\phi_{0}^{\dagger}(P) \phi_{0}(P)\right]^{2}\right. \\
& +2 \phi_{0}^{\dagger}(P) \phi_{0}(P)\left[\phi_{0}^{\dagger}(P) \chi(P)+\chi^{\dagger}(P) \phi_{0}(P)\right] \\
& \left.+2 \phi_{0}^{\dagger}(P) \phi_{0}(P)\left[\phi_{0}^{\dagger}(P) \phi^{\prime}(P)+\phi^{\prime \dagger}(P) \phi_{0}(P)\right]+\ldots\right\}
\end{aligned}
$$


where $\cdots$ indicate terms of higher powers of $\chi$ and $\phi^{\prime}$. We would like to emphasize here that the second and third terms of the right hand side of (5.4) are in general nonzero. Indeed, for the color flavor locked condensate (3.21), at $N_{c}=N_{f}=N$

$$
\begin{aligned}
\phi_{f_{1} f_{2}}^{c_{1} c_{2}}(P) & =\frac{1}{2}\left[B_{1}(P)-B_{2}(P)\right]\left(\delta_{f_{1}}^{c_{1}} \delta_{f_{2}}^{c_{2}}-\delta_{f_{1}}^{c_{2}} \delta_{f_{2}}^{c_{1}}\right) \\
\chi_{f_{1} f_{2}}^{c_{1} c_{2}}(P) & =\frac{1}{2}\left[B_{1}(P)+B_{2}(P)\right]\left(\delta_{f_{1}}^{c_{1}} \delta_{f_{2}}^{c_{2}}+\delta_{f_{1}}^{c_{2}} \delta_{f_{2}}^{c_{1}}\right),
\end{aligned}
$$

we find that

$$
\begin{aligned}
& \operatorname{Tr} \phi^{\dagger}(P) \phi(P) \phi^{\dagger}(P) \chi(P)= \\
& =\frac{N\left(N^{2}-1\right)(N-2)}{16}\left|B_{1}(P)-B_{2}(P)\right|^{2}\left[B_{1}(P)-B_{2}(P)\right]^{*}\left[B_{1}(P)+B_{2}(P)\right]
\end{aligned}
$$

which is nonzero for $N>2$. By combining (4.36) and (5.4), we derive an expression for the Ginzburg-Landau free energy functional for a homogeneous system,

$$
\begin{aligned}
\Gamma(\mathcal{B}) & =\frac{k_{B} T_{C}}{2} \sum_{P^{\prime} P}<P^{\prime}\left|\mathcal{M}_{A}\right| P>\operatorname{Tr} \phi_{0}^{\dagger}\left(P^{\prime}\right) \phi_{0}(P) \\
& +\frac{k_{B} T_{C}}{2} \sum_{P}\left[\nu_{n}^{2}+(p-\mu)^{2}\right] \operatorname{Tr}\left[\phi^{\prime \dagger}(P) \phi^{\prime}(P)+\chi^{\dagger}(P) \chi(P)\right] \\
& +\frac{k_{B} T_{C}}{4} \sum_{P}\left(\nu_{n}^{2}+(p-\mu)^{2}\right]^{2} \operatorname{Tr}\left\{\left[\phi_{0}^{\dagger}(P) \phi_{0}(P)\right]^{2}\right. \\
& +2 \phi_{0}^{\dagger}(P) \phi_{0}(P)\left[\phi_{0}^{\dagger}(P) \chi(P)+\chi^{\dagger}(P) \phi_{0}(P)\right] \\
& \left.+2 \phi_{0}^{\dagger}(P) \phi_{0}(P)\left[\phi_{0}^{\dagger}(P) \phi^{\prime}(P)+\phi^{\prime \dagger}(P) \phi_{0}(P)\right]+\ldots\right\}
\end{aligned}
$$

where a zeroth order approximation in $g$ has been made for the coefficient in front of the non-pairing modes of the quadratic terms. Minimization with respect to $\chi$ yields

$$
\begin{aligned}
\chi_{f_{1} f_{2}}^{c_{1} c_{2}}(P) & =-\frac{1}{4}\left[\nu_{n}^{2}+(p-\mu)^{2}\right]\left\{\left[\phi_{0}(P) \phi_{0}^{\dagger}(P) \phi_{0}(P)\right]_{f_{1} f_{2}}^{c_{1} c_{2}}+\left[\phi_{0}(P) \phi_{0}^{\dagger}(P) \phi_{0}(P)\right]_{f_{1} f_{2}}^{c_{2} c_{1}}\right. \\
& \left.+\left[\phi_{0}(P) \phi_{0}^{\dagger}(P) \phi_{0}(P)\right]_{f_{2} f_{1}}^{c_{1} c_{2}}+\left[\phi_{0}(P) \phi_{0}^{\dagger}(P) \phi_{0}(P)\right]_{f_{2} f_{1}}^{c_{2} c_{1}}\right\}
\end{aligned}
$$

and its contribution to the free energy is of the order of $\left|\phi_{0}\right|^{6}$. Below the transition temperature, $\phi_{0}(P) \sim\left(T_{C}-T\right)^{\frac{1}{2}}$ for $T_{C}-T<<T_{C}$ and the bulk condensate energy $\sim\left(T_{C}-T\right)^{2}$. We also notice the difference from the $T=0$ case, where the symmetric condensate is suppressed relatively to the antisymmetric one by a factor of order $g$ [16]. On the other hand, the induced color-symmetric condensate, $\chi \sim\left(T_{C}-T\right)^{\frac{3}{2}}$ and its contribution to the free energy $\sim\left(T_{C}-T\right)^{3}$. The same is true for the non-pairing modes in the color-antisymmetric component, $\phi^{\prime}(P)$. Maintaining only the pairing mode, we may write

$$
\phi(P) \simeq \phi_{0}(P)=\sqrt{6} \Psi u(P)
$$


where $u(P)$ is the eigenfunction of the pairing mode, (4.29), and $\Psi$ is a constant $N_{c} N_{f} \times$ $N_{c} N_{f}$ matrix with the same symmetry of the indices as $\phi(P)$.

It follows from the expression (4.29) for $u(P),(4.28)$ for $h(P)$ and the eigenvalue (4.32) that

$$
\Gamma(\mathcal{B})=\Omega\left[\frac{a}{2} \operatorname{Tr} \Psi^{\dagger} \Psi+\frac{b}{4} \operatorname{Tr}\left(\Psi^{\dagger} \Psi\right)^{2}\right] .
$$

with

$$
a=\frac{48 \pi^{2}}{7 \zeta(3)} k_{B}^{2} T_{C}\left(T-T_{C}\right)
$$

and

$$
b=\frac{576 \pi^{4}}{7 \zeta(3)}\left(\frac{k_{B} T_{C}}{\mu}\right)^{2} .
$$

Including both helicities, we obtain the total Ginzburg-Landau free energy of a homogeneous system with an even parity for the ground state

$$
\Gamma_{\text {total }}\left(\mathcal{B}_{L}, \mathcal{B}_{R}\right)=\Omega\left[a \operatorname{Tr} \Psi^{\dagger} \Psi+\frac{b}{2} \operatorname{Tr}\left(\Psi^{\dagger} \Psi\right)^{2}\right]
$$

We conclude this section with some details for the realistic case of $N_{c}=N_{f}=3$. With even parity the $9 \times 9$ matrix $\Psi$ may be related to a $3 \times 3$ matrix via

$$
\Psi_{f_{1} f_{2}}^{c_{1} c_{2}}=\epsilon^{c_{1} c_{2} c} \epsilon_{f_{1} f_{2} f} \Phi_{f}^{c}
$$

The trace of $\Psi$ 's may be related to the trace of $\Phi$ 's. Explicitly,

$$
\begin{aligned}
\operatorname{Tr} \Psi^{\dagger} \Psi & =4 \operatorname{tr} \Phi^{\dagger} \Phi \\
\operatorname{Tr}\left(\Psi^{\dagger} \Psi\right)^{2} & =2\left[\left(\operatorname{tr} \Phi^{\dagger} \Phi\right)^{2}+\operatorname{tr}\left(\Phi^{\dagger} \Phi\right)^{2}\right] .
\end{aligned}
$$

Consequently, the Ginzburg-Landau free energy reduces to

$$
\Gamma_{\text {total }}=\Omega\left\{4 a \operatorname{tr} \Phi^{\dagger} \Phi+b\left[\left(\operatorname{tr} \Phi^{\dagger} \Phi\right)^{2}+\operatorname{tr}\left(\Phi^{\dagger} \Phi\right)^{2}\right]\right\}
$$

where $a$ and $b$ are given in (5.11) and (5.12) at weak coupling with $a<0$ for $T<T_{C}$ and $b>0$. The minimum free energy below $T_{C}$ corresponds to

$$
\Phi^{\dagger} \Phi=\frac{|a|}{2 b}
$$

i.e. $\Phi$ is proportional to an unitary matrix. By a flavor and baryon number rotation, it can always be brought into an unit matrix, which leads to the standard expression of the color-flavor locked condensate with

$$
\Psi_{f_{1} f_{2}}^{c_{1} c_{2}}=\sqrt{\frac{|a|}{2 b}}\left(\delta_{f_{1}}^{c_{1}} \delta_{f_{2}}^{c_{2}}-\delta_{f_{1}}^{c_{2}} \delta_{f_{2}}^{c_{1}}\right) .
$$


The corresponding condensate energy is

$$
\left(\Gamma_{\text {total }}\right)_{\min }=-6 \frac{a^{2}}{b} \Omega=-\frac{12 \mu^{2}}{7 \zeta(3)} k_{B}^{2} T_{C}^{2}\left(\frac{T_{C}-T}{T_{C}}\right)^{2} \Omega
$$

We have thus proven that the color-flavor locked condensate remains energetically favored near $T_{c}$. Using (5.8) and (5.9), the induced sextet order parameter reads

$$
\chi_{f_{1} f_{2}}^{c_{1} c_{2}}(P)=-3 \sqrt{3}\left(\frac{|a|}{b}\right)^{\frac{3}{2}}\left[\nu_{n}^{2}+(p-\mu)^{2}\right] u^{3}(P)\left(\delta_{f_{1}}^{c_{1}} \delta_{f_{2}}^{c_{2}}+\delta_{f_{1}}^{c_{2}} \delta_{f_{2}}^{c_{1}}\right)
$$

and it contributes to the condensate energy a term

$$
\Delta \Gamma_{\text {min }}=-\frac{558 \zeta(5)}{343 \zeta^{3}(3)} \mu^{2} k_{B}^{2} T_{C}^{2}\left(\frac{T_{C}-T}{T_{C}}\right)^{3},
$$

which is smaller than (5.19) by a factor $\frac{\left(T_{C}-T\right)}{T_{C}}$. The existence of the sextet order parameter is evident from the composition rule $\mathbf{3} \times \overline{\mathbf{3}} \times \overline{\mathbf{3}}=\overline{\mathbf{3}}+\overline{\mathbf{3}}+\mathbf{6}+\mathbf{1 5}$ of the color $S U(3)$ representations and its conjugate for the flavor $S U(3)$ representations. The sextet correction (5.21) may be compared with the correction due to the constraint of color neutrality discussed in [15]. The latter is suppressed by a factor of $\left(\frac{k_{B} T_{c}}{\mu}\right)^{2} \ln \frac{\mu}{k_{B} T_{c}}$ relative to (5.19). If $\frac{T_{c}-T}{T} \geq\left(\frac{k_{B} T_{c}}{\mu}\right)^{2} \ln \frac{\mu}{k_{B} T_{c}}$, the sextet correction will be larger in magnitude.

\section{Kinetic Energy and Gauge Coupling.}

An important application of the Ginzburg-Landau theory lies in systems which include inhomogeneous condensates, such as domain walls and vortex filaments. To incorporate the spatial variation of the condensate, a nonzero total momentum is introduced to the di-quark propagator. As we shall see below, the characteristic momentum corresponds to the inverse of the coherence length, which is much longer than the thermal wavelength of the system and diverges at $T_{c}$. Therefore, the momentum dependence of the di-quark propagator will be treated perturbatively while the dependence of the quartic coefficient will enter only through the constraint of the overall momentum conservation.

A non-zero total momentum $\vec{k}$ will modify both the disconnected and the connected part of the di-quark propagator. The spectral problem (4.16) is replaced by

$$
D_{\vec{k}}^{-1}(P) u_{\vec{k}}(P)+\frac{1}{\beta \Omega} \sum_{P^{\prime}} \Gamma_{A, \vec{k}}\left(P \mid P^{\prime}\right) u_{\vec{k}}\left(P^{\prime}\right)=E u_{\vec{k}}(P)
$$


where we have appended the subscript $\vec{k}$ to indicate the dependence on the total momentum while the relative 4-Euclidean momentum remains denoted by $P$. Thus the 4-Euclidean momentum of each quark in a pair is $\left(\frac{\vec{k}}{2} \pm \vec{p}, \mp \nu_{n}\right)$. The homogeneous case considered in section 3 corresponds to the special case $\vec{k}=0$. As it was shown in section 3 , the quark self-energy may be omitted to the leading order of the interaction, except in the determination of the transition temperature, $D_{\vec{k}}^{-1}(P) \simeq\left(i \nu_{n}-p_{+}+\mu\right)\left(-i \nu_{n}-p_{-}+\mu\right)$ with $p_{ \pm}=\left|\frac{\vec{k}}{2} \pm \vec{p}\right|$. The Fredholm equation (4.21) becomes

$$
h_{\vec{k}}(P)=\frac{\lambda^{2}}{\beta \Omega} \sum_{P^{\prime}} K_{E, \vec{k}}\left(P \mid P^{\prime}\right) h_{\vec{k}}\left(P^{\prime}\right)
$$

with

$$
K_{E, \vec{k}}\left(P^{\prime} \mid P\right)=-\frac{\bar{\Gamma}_{A, \vec{k}}\left(P^{\prime} \mid P\right)}{\left(i \nu_{n}-p_{+}+\mu\right)\left(-i \nu_{n}-p_{-}+\mu\right)-E} .
$$

The expansion (4.23) is supplemented by more terms

$$
K_{E, \vec{k}}\left(P \mid P^{\prime}\right)=K\left(P \mid P^{\prime}\right)+\delta K\left(P \mid P^{\prime}\right)+\delta^{\prime} K\left(P \mid P^{\prime}\right)+\delta^{\prime \prime} K\left(P \mid P^{\prime}\right)
$$

with

$$
\begin{array}{r}
\delta^{\prime} K\left(P \mid P^{\prime}\right)=-K\left(P \mid P^{\prime}\right)\left[-\frac{i \nu_{n}}{\nu_{n}^{2}+(p-\mu)^{2}} \hat{p} \cdot \vec{k}-\frac{1}{4} \frac{3 \nu_{n}^{2}-(p-\mu)^{2}}{\left(\nu_{n}^{2}+(p-\mu)^{2}\right)^{2}}(\hat{p} \cdot \vec{k})^{2}\right. \\
\left.-\frac{(p-\mu)}{4 p} \frac{\vec{k}^{2}-(\vec{k} \hat{p})^{2}}{\nu_{n}^{2}+(p-\mu)^{2}}\right]
\end{array}
$$

while the term $\delta^{\prime \prime} K\left(P \mid P^{\prime}\right)$ arises entirely due to the momentum correction of the vertex function. The corresponding eigenvalue condition (4.24) is replaced by

$$
1=\lambda^{-2}(T, \mu, 0)+c(T, \mu) E+c^{\prime}(T, \mu) \vec{k}^{2}
$$

where $c^{\prime}(T, \mu)$ is computed perturbatively, like $c(T, \mu)$ of (4.31). The first order perturbation yields the result

$$
c^{\prime}\left(T_{C}, \mu\right)=-\frac{7 \zeta(3)}{24 \pi^{2} k_{B}^{2} T_{C}^{2} \ln \frac{1}{\epsilon_{C}}}=-\frac{1}{6} c\left(T_{C}, \mu\right) .
$$

This contribution arises exclusively from the second term inside the bracket of (6.5), while the third term contributes a term smaller by a factor whose order is less than $\left(\frac{k_{B} T_{c}}{\mu}\right)^{2} \ln \frac{\mu}{k_{B} T_{c}}$. The second order perturbation of the first term inside the bracket of (6.5) contributes to $c^{\prime}(T, \mu)$ a term that is smaller than (6.7) by a factor of order $g$. Finally lets comment that the contribution of the term $\delta^{\prime \prime} K\left(P \mid P^{\prime}\right)$ is analyzed in Appendix $\mathrm{B}$, where we show that its contribution to $c^{\prime}\left(T_{c}, \mu\right)$ is comparable with that of the third 
term inside the bracket of (6.5) and therefore can be ignored. Finally, the eigenvalue of the pairing mode with a small total momentum becomes

$$
E \simeq \frac{8 \pi^{2}}{7 \zeta(3)} k_{B}^{2} T_{C}\left(T-T_{C}\right)+\frac{1}{6} \vec{k}^{2}
$$

Therefore, the typical momentum of the condensate is $\sim k_{B} T_{C} \sqrt{\frac{T_{C}-T}{T_{C}}}$, whose inverse corresponds to the coherence length. For a condensate consisting of superpositions of different $\vec{k}$ 's, i.e.

$$
B(P)=\sqrt{6} \sum_{\vec{k}} \Psi_{\vec{k}} u_{\vec{k}}(P)
$$

the Ginzburg-Landau functional becomes

$$
\Gamma=\frac{1}{2} \sum_{\vec{k}}\left(\vec{k}^{2}+a\right) \operatorname{Tr} \Psi_{\vec{k}}^{\dagger} \Psi_{\vec{k}}+\frac{1}{4} b \sum_{\overrightarrow{k_{1}} \overrightarrow{k_{2}} \overrightarrow{k_{1}} \vec{k}_{k_{2}}^{\prime}} \operatorname{Tr} \Psi_{\overrightarrow{k_{1}}}^{\dagger} \Psi_{\overrightarrow{k_{1}}} \Psi_{\overrightarrow{k_{2}}}^{\dagger}, \Psi_{\overrightarrow{k_{2}}} \delta_{\overrightarrow{k_{1}}+\overrightarrow{k_{2}}, \overrightarrow{k_{1}}+\overrightarrow{k_{2}}}
$$

By transforming the condensate from the momentum space into the coordinate space via

$$
\Psi(\vec{r})=\frac{1}{\sqrt{\Omega}} \sum_{\vec{k}} \Psi_{\vec{k}} e^{i \vec{k} \vec{r}}
$$

and including both helicities with an even parity ground state we obtain the conventional form of the Ginzburg-Landau free energy

$$
\Gamma_{\text {total }}=\int d^{3} \vec{r}\left[\operatorname{Tr} \vec{\nabla} \Psi^{\dagger} \vec{\nabla} \Psi+a \operatorname{Tr} \Psi^{\dagger} \Psi+\frac{1}{2} b \operatorname{Tr}\left(\Psi^{\dagger} \Psi\right)^{2}\right]
$$

It is instuctive to compare the Ginzburg-Landau free energy (6.12) with that of the ordinary BCS model. On setting $v_{F}=1$ for the latter and scaling $\Psi_{\vec{k}} \mapsto \sqrt{2 k_{F}} \Psi_{\vec{k}}$, the free energy as a polynomial in $\Psi_{\vec{k}}$ contains identical coefficients as $(3.21)$.

It remains to couple the order parameter to the classical Yang-Mills color gauge field and to the electromagnetic field. While the coupling structure is dictated by diagrams, we shall follow a different route by replacing the gradient in (6.12) with the gauge covariant derivative. As the final result ought to be gauge invariant, such an approach is unambigious. Let $\vec{A}=\vec{A}^{l} T^{l}$ denote the classical vector potential of the $S U\left(N_{c}\right)$ color gauge field and $\overrightarrow{\mathcal{A}}$ that of the electromagnetic field, of which the associated $U(1)$ rotation is a subgroup of the flavor $S U\left(N_{f}\right)$ group. The gauge covariant derivative acting on a single quark field is

$$
\left(\vec{D} \psi^{c}\right)_{f}=\vec{\nabla} \psi_{f}^{c}-i g \vec{A}^{c c^{\prime}} \psi_{f}^{c^{\prime}}-i e q_{f} \psi_{f}^{c^{\prime}}
$$


with $q_{f}$ being the charge number of the $f$-th flavor. From here on, only the repeated color indices in (6.13) are summed. Since the order parameter behaves like a direct product of two quark fields, the gauge covariant derivative of $\Psi$ reads

$$
(\vec{D} \Psi)_{f_{1} f_{2}}^{c_{1} c_{2}}=\vec{\nabla} \Psi_{f_{1} f_{2}}^{c_{1} c_{2}}-i g \vec{A}^{c_{1} c^{\prime}} \Psi_{f_{1} f_{2}}^{c^{\prime} c_{2}}-i g \vec{A}^{c_{2} c^{\prime}} \Psi_{f_{1} f_{2}}^{c_{1} c^{\prime}}-i e\left(q_{f_{1}}+q_{f_{2}}\right) \overrightarrow{\mathcal{A}} \Psi_{f_{1} f_{2}}^{c_{1} c_{2}}
$$

By replacing $\vec{\nabla} \Psi$ of (6.12) with $\vec{D} \Psi$ and including the energy of the pure Yang-Mills part, we obtain the gauge invariant Ginzburg-Landau free energy functional of an inhomogeneous superconductor

$$
\Gamma_{\text {total }}=\int d^{3} \vec{r}\left[\frac{1}{2} F_{i j}^{l} F_{i j}^{l}+\frac{1}{2}(\vec{\nabla} \times \overrightarrow{\mathcal{A}})^{2}+\operatorname{Tr}(\vec{D} \Psi)^{\dagger}(\vec{D} \Psi)+a \operatorname{Tr} \Psi^{\dagger} \Psi+\frac{1}{2} b \operatorname{Tr}\left(\Psi^{\dagger} \Psi\right)^{2}\right]
$$

It is easy to verify that the free energy functional is invariant under the local gauge transformations

$$
\begin{aligned}
\Psi_{f_{1} f_{2}}^{c_{1} c_{2}} & \mapsto u^{c_{1} c_{1}^{\prime}} u^{c_{2} c_{2}^{\prime}} \Psi_{f_{1} f_{2}}^{c_{1}^{\prime} c_{2}^{\prime}} e^{-i\left(q_{f_{1}}+q_{f_{2}}\right) \alpha} \\
\vec{A} & \mapsto u \vec{A} u^{\dagger}+\frac{i}{g} u \vec{\nabla} u^{\dagger} \\
\overrightarrow{\mathcal{A}} & \mapsto \overrightarrow{\mathcal{A}}-\frac{1}{e} \vec{\nabla} \alpha
\end{aligned}
$$

The equation of motion follows from the free energy functional by applying the variational principle.

Finally, let us explore some details for the realistic case of $N_{c}=N_{f}=3$. It follows from (6.13) that

$$
\left(\vec{D} \Psi^{c_{1} c_{2}}\right)_{f_{1} f_{2}}=\epsilon^{c_{1} c_{2} c} \epsilon_{f_{1} f_{2} f}(\vec{D} \Phi)_{f}^{c}
$$

with

$$
(\vec{D} \Phi)_{f}^{c}=\vec{\nabla} \Phi_{f}^{c}-i g \overrightarrow{\vec{A}}^{c c^{\prime}} \Phi_{f}^{c^{\prime}}-i e Q_{f} \overrightarrow{\mathcal{A}} \Phi_{f}^{c}
$$

where $\overrightarrow{\vec{A}}=\vec{A}^{l} \bar{T}^{l}$ with $\bar{T}^{l}=-T^{l *}$ being the generator of the $\overline{\mathbf{3}}$ representation, and $Q_{f}=$ $q_{f_{1}}+q_{f_{2}}$ and $f f_{1} f_{2}$ represent a cyclic permutation of 1,2,3. Arranging the flavor index in the conventional order of $u, d, s$ we find the diagonal electric charge matrix

$$
Q=\operatorname{diag}\left(\frac{2}{3},-\frac{1}{3},-\frac{1}{3}\right)=\frac{2}{\sqrt{3}} \bar{T}^{8} .
$$

Here we have adapted an expression of $T^{l}$ that differs from the standard one by a cyclic permutation of rows (columns), in which $\bar{T}^{8}=\frac{1}{2 \sqrt{3}} \operatorname{diag}(2,-1,-1)$. The equation (6.18) takes the matrix form

$$
\vec{D} \Phi=\vec{\nabla} \Phi-i g \overrightarrow{\bar{A}} \Phi-i e q \overrightarrow{\mathcal{A}} \Phi \bar{T}^{8}
$$

with $q=-\frac{2}{\sqrt{3}} e$. On noting that $\Phi=\Phi_{0}+\bar{T}^{l} \Phi_{l}$, we have the decomposition

$$
\vec{D} \Phi=(\vec{D} \Phi)_{0}+\bar{T}^{l} \vec{D} \Phi_{l}
$$


with $(\vec{D} \Phi)_{0}=\frac{1}{3} \operatorname{tr}(\vec{D} \Phi)$ and $\vec{D} \Phi_{l}=2 \operatorname{tr} \bar{T}^{l} \vec{D} \Phi$, and its contribution to the free energy $(6.15)$

$$
\operatorname{tr}(\vec{D} \Phi)^{\dagger}(\vec{D} \Phi)=3(\vec{D} \Phi)_{0}^{*}(\vec{D} \Phi)_{0}+\frac{1}{2}(\vec{D} \Phi)_{l}^{*}(\vec{D} \Phi)_{l}
$$

Now we divide the field variables into two groups, the first group contains $\Phi_{0}, \Phi_{8}, \overrightarrow{A_{8}}, \overrightarrow{\mathcal{A}}$, while the second one includes $\Phi_{l}, \vec{A}^{l}$ with $l \neq 0,8$. A closer inspection of the structure of $\vec{D} \Phi$ reveals the absence terms in the free energy (6.15) that are linear in the variables of the second group. Therefore a solution of the equation of motion exists in which only the fields of the first group acquire non-zero values. In what follows, we shall focus on this special case.

By setting $\Phi_{l}=\vec{A}^{l}=0$ for $l \neq 0,8$ and transforming the remaining variables

$$
\begin{aligned}
& \phi=\Phi_{0}+\frac{1}{\sqrt{3}} \Phi^{8}, \quad \chi=\sqrt{2}\left(\Phi_{0}-\frac{1}{2 \sqrt{3}} \Phi^{8}\right) \\
& \vec{V}=\vec{A}^{8} \cos \theta+\overrightarrow{\mathcal{A}} \sin \theta \quad \overrightarrow{\mathcal{V}}=-\vec{A}^{8} \sin \theta+\overrightarrow{\mathcal{A}} \cos \theta
\end{aligned}
$$

with $\tan \theta=-\frac{2 e}{\sqrt{3} g}$ we arrive at the following expression for the Ginzburg-Landau free energy functional (6.15)

$$
\begin{aligned}
\Gamma_{\text {total }}=\int d^{3} \vec{r}( & \frac{1}{2}(\vec{\nabla} \times \overrightarrow{\mathcal{V}})^{2}+\frac{1}{2}(\vec{\nabla} \times \vec{V})^{2}+|(\vec{\nabla}-2 i \lambda \vec{V}) \phi|^{2}+|(\vec{\nabla}+i \lambda \vec{V}) \chi|^{2} \\
& \left.+4 a\left(|\phi|^{2}+|\chi|^{2}\right)+b\left[\left(|\phi|^{2}+|\chi|^{2}\right)^{2}+|\phi|^{4}+\frac{1}{2}|\chi|^{4}\right]\right),
\end{aligned}
$$

with $\lambda=\frac{1}{2 \sqrt{3}} \sqrt{g^{2}+\frac{4}{3} e^{2}}$. The transformation of the gauge potentials in (6.23) is nothing but the transformation performed in [3], [21], [22] that selects out the unbroken $U(1)$ gauge potential $\vec{V}$ in the color-flavor locked phase. The $\overrightarrow{\mathcal{V}}$ field remains decoupled from the condensate in the presence of a nonzero $\Phi^{8}$ and can be set to zero as a special solution. The condition for the color-flavor locking (that the $3 \times 3$ matrix is proportional to an unitary matrix),

$$
|\chi|^{2}=2|\phi|^{2}
$$

which corresponds to the free energy minimum in the bulk, cannot be imposed everywhere because of the nonzero values of $\vec{\nabla} \phi, \vec{\nabla} \chi$ and $\vec{V}$, as it is evident from the example of a vortex filament and a domain wall which are analyzed in the appendix $\mathrm{C}$. In another words, in the presence of an inhomogeneity and of a non zero gauge potential, the unlocked condensate, which is an octet, under a simultaneous color-flavor rotation mixes with the locked condensate. This situation may be compared with the $s-d$ wave mixture in the core region of a vortex filament of a cuprite superconductor [23]. 


\section{Concluding Remarks.}

In this work, we have derived the Ginzburg-Landau free energy functional of the color superconductivity from the fundamental QCD action by means of normal phase thermal diagrams. We have also addressed the issue of the induced color symmetric component of the condensate near $T_{C}$ and the interplay between the color-flavor locked condensate and the unlocked one in the presence of a gauge potential. Though the expressions of the Ginzburg-Landau coefficients were derived in the weak coupling approximation, which is valid in the ultra-high baryon density regime, we expect that many qualitative features, such as the color-flavor locking, the mixture of the color-symmetric condensate and the coupling among the different types of condensates and the gauge potential, to survive in the regime of realistic baryon density, e.g., the baryon density achieved at RHIC or that inside of a neutron star.

As the chemical potential is lowered, higher order corrections become more important. There are two types of higher order terms, which constitute a hierarchy of smallness, those which result from higher powers of the coupling constant $g$ and those that arise from the curvature of the density of states at the Fermi level. It follows from (1) that

$g \sim \ln ^{-1} \mu / k_{B} T_{C}$, while the latter corrections come in powers of $\left(k_{B} T_{C} / \mu\right)^{2}$. Therefore the corrections due to the higher order thermal diagrams are far more important. In view of the $2 \pi$ suppression of the integral over loop momenta inside higher order diagrams (before the combinatorial factor dominating the magnitude), the numerical values of the Ginzburg-Landau coefficients we derived may still serve as a reasonable approximation for a realistic high baryon density.

\section{Acknowlegments.}

We would like like to thank D. T. Son for raising the issue of Ginzburg-Landau theory which motivated this reasearch. This work is supported in part by US Department of Energy, contract number DE-FG02-91ER40651-TASKB. 


\section{Appendix A.}

In this appendix, we shall illustrate our methodology by rederiving the GinzburgLandau free energy for the BCS model of the system of nonrelativistic electrons. The model Hamiltonian is given by

$$
H=\sum_{\vec{p} s} \xi_{\vec{p}} a_{\vec{p} s}^{\dagger} a_{\vec{p} s}-\frac{g}{\Omega} \sum_{\vec{p}, \vec{p}^{\prime}} \theta\left(\omega_{D}-\left|\xi_{\vec{p}}\right|\right) \theta\left(\omega_{D}-\left|\xi_{\vec{p}^{\prime}}\right|\right) a_{\vec{p} \uparrow}^{\dagger} a_{-\vec{p} \downarrow}^{\dagger} a_{-\vec{p}^{\prime} \downarrow} a_{\vec{p}^{\prime} \uparrow}
$$

where $\xi_{\vec{p}}=\frac{p^{2}}{2 m}-\mu, m$ the electron mass and $\mu$ the chemical potential. The subscript $s$ labels the spin state, $g>0$ is the pairing coupling and $\omega_{D}$ denotes the Debye frequency. The path integral expression of the free energy at a temperature $T$ reads

$$
\exp \left(-\frac{F}{k_{B} T}\right)=C \int \prod_{\vec{p} s} d a_{\vec{p} s} d \bar{a}_{\vec{p} s} e^{-S_{E}(a, \bar{a})}
$$

where the Euclidean action

$$
\begin{aligned}
S_{E}(a, \bar{a}) & =\sum_{P s}\left(-i \nu_{n}+\xi_{\vec{p}}\right) \bar{a}_{P s} a_{P s} \\
& -\frac{g}{\beta \Omega} \sum_{P_{+} P_{-}, P_{+}^{\prime}, P_{-}^{\prime}} \theta\left(\omega_{D}-\left|\xi_{\vec{p}}\right|\right) \theta\left(\omega_{D}-\left|\xi_{\vec{p}^{\prime}}\right|\right) a_{P_{+} \uparrow}^{\dagger} a_{P_{-} \downarrow}^{\dagger} a_{P_{-}^{\prime} \downarrow} a_{P_{+}^{\prime} \uparrow} \delta_{n_{+}+n_{-}, n_{+}^{\prime}+n_{-}^{\prime}},
\end{aligned}
$$

where $P=\left(\vec{p},-\nu_{n}\right)$ is the Euclidean four momentum and $P_{ \pm}=( \pm \vec{p}, \mp \nu)$. The Matsubara energy $\nu_{n}=2 \pi n k_{B} T$ with $n= \pm \frac{1}{2}, \pm \frac{3}{2}, \ldots$. Following the steps outlined in section 2, we add to $S_{E}$ a triggering term

$$
\Delta S=\sum_{P} \theta\left(\omega_{D}-\left|\xi_{\vec{p}}\right|\right)\left[J^{*}(P) a_{-P \downarrow} a_{P \uparrow}+J(P) a_{P \uparrow}^{\dagger} a_{-P \downarrow}^{\dagger}\right],
$$

which gives rise to an increment $W(J)$ of the free energy and induces an order parameter

$$
B(P)=\frac{\delta W}{\delta J^{*}(P)}
$$

The Legendre transformation of $W(J)$,

$$
\Gamma(B)=\Gamma_{2}+\Gamma_{4}+\ldots
$$

is the Ginzburg-Landau functional and determines the order parameter at equilibrium when the triggering term (9.4) is removed, with

$$
\Gamma_{2}=\sum_{P, P^{\prime}} B^{*}(P)<P|\mathcal{M}| P^{\prime}>B\left(P^{\prime}\right)
$$


and

$$
\Gamma_{4}=\frac{1}{2} \sum_{P_{1}, P_{2}, P_{1}^{\prime}, P_{2}^{\prime}} B^{*}\left(P_{1}\right) B^{*}\left(P_{2}\right)<P_{1} P_{2}|\mathcal{G}| P_{1}^{\prime} P_{2}^{\prime}>B\left(P_{1}^{\prime}\right) B\left(P_{2}^{\prime}\right) .
$$

The coefficient $<P|\mathcal{M}| P^{\prime}>$ is the inverse di-electron propagator, given by

$$
<P|\mathcal{M}| P^{\prime}>=\left(\nu_{n}^{2}+\xi_{\vec{p}}^{2}\right) \delta_{P P^{\prime}}-\frac{g}{\beta \Omega} \theta\left(\omega_{D}-\left|\xi_{\vec{p}}\right|\right) \theta\left(\omega_{D}-\left|\xi_{\vec{p}^{\prime}}\right|\right)
$$

where the second term on the right hand side represents the leading order 2PI vertex in accordance with the Hamiltonian (9.1).

The eigenvalues and eigenfunctions of $\mathcal{M}$ are determined by

$$
\left(\nu_{n}^{2}+\xi_{\vec{p}}^{2}\right) u(P)-\frac{g}{\beta \Omega} \theta\left(\omega_{D}-\left|\xi_{\vec{p}}\right|\right) \sum_{\vec{p}^{\prime}} \theta\left(\omega_{D}-\left|\xi_{\vec{p}^{\prime}}\right|\right) u\left(P^{\prime}\right)=E u(P) .
$$

On writing

$$
A=\frac{g}{\beta \Omega} \sum_{P^{\prime}} \theta\left(\omega_{D}-\left|\xi_{\vec{p}}\right|\right) u(P)
$$

we have

$$
u(P)=\frac{A}{\nu_{n}^{2}+\xi_{\vec{p}}^{2}-E} .
$$

Substituting (9.12) back to (9.11), we obtain the secular equation for $E$

$$
1=\frac{g}{\beta \Omega} \sum_{P} \frac{\theta\left(\omega_{D}-\left|\xi_{\vec{p}}\right|\right)}{\nu_{n}^{2}+\xi_{\vec{p}}^{2}-E}
$$

The pairing mode corresponds to the eigenvalue that vanishes at $T_{C}$. For $T$, sufficiently close to $T_{C}$, we may expand the r. h. s. of (9.13) to first order in $E$, i.e.

$$
\begin{aligned}
1 & =\frac{g}{\beta \Omega} \sum_{P} \frac{\theta\left(\omega_{D}-\left|\xi_{\vec{p}}\right|\right)}{\nu_{n}^{2}+\xi_{\vec{p}}^{2}}+\frac{g E}{\beta \Omega} \sum_{P} \frac{\theta\left(\omega_{D}-\left|\xi_{\vec{p}}\right|\right)}{\left(\nu_{n}^{2}+\xi_{\vec{p}}^{2}\right)^{2}} \\
& =\frac{g k_{F}^{2}}{2 \pi^{2} v_{F}}\left(\ln \frac{2 \omega_{D}}{\pi k_{B} T}+\gamma\right)+g \frac{7 \zeta(3) k_{F}^{2}}{16 \pi^{2} v_{F}\left(k_{B} T\right)^{2}} E
\end{aligned}
$$

with $k_{F}$ the Fermi momentum, $v_{F}$ the Fermi velocity and $\gamma$ the Euler constant. At the transition temperature $T=T_{c}$ the following relation holds,

$$
1=\frac{g k_{F}^{2}}{2 \pi^{2} v_{F}}\left(\ln \frac{2 \omega_{D}}{\pi k_{B} T_{C}}+\gamma\right)
$$

and $E=0$ according to (9.14). In the neighbourhood of $T_{C}$, we have

$$
E=\frac{8 \pi^{2}}{7 \zeta(3)} k_{B}^{2} T_{C}\left(T-T_{C}\right)
$$


All other modes are nonpairing with eigenvalues $E_{l}=\nu_{n}^{2}+\xi_{\vec{p}}^{2}+O(g)$. Expanding the order parameter according to the eigenfunctions of $\mathcal{M}$, i.e.

$$
B(P)=\Psi u(P)+B^{\prime}(P)
$$

with $u(P)$ the eigenfunction of the pairing mode and $\frac{1}{\beta \Omega} \sum_{P} u^{\dagger}(P) B^{\prime}(P)=0$, we find

$$
\Gamma_{2}=E \Psi^{*} \Psi+\sum_{P}\left(\nu_{n}^{2}+\xi_{\vec{p}}^{2}\right) B^{\prime *}(P) B^{\prime}(P)
$$

To calculate the quartic term, we switch off the interaction and obtain the quartic term of $J$ in $W(J)$. Then we amputate it with four di-electron propagators. The result reads

$$
\Gamma_{4}=\frac{|\Psi|^{4}}{2 \beta \Omega} \sum_{P}\left(\nu_{n}^{2}+\xi_{\vec{p}}\right)^{2}|u(P)|^{4}+\ldots
$$

where the terms in ... contain nonpairing modes and are at most $\sim|\Psi|^{3}$ as $T \mapsto T_{C}$. Neglecting these modes, we find

$$
\Gamma(\Psi)=\Omega\left[\frac{8 \pi^{2} k_{B}^{2} T_{C}}{7 \zeta(3)}\left(T-T_{C}\right)|\Psi|^{2}+\frac{4 \pi^{4}\left(k_{B} T_{C}\right)^{2}}{7 \zeta(3) k_{F}^{2}}|\Psi|^{4}\right]
$$

If a small momentum $\vec{k}$ is introduced, only the eigenvalue of the pairing mode is modified by an amount comparable to itself. In order to determine $E$ in this case, we observe that (9.13) is replaced by

$$
1=\frac{g}{\beta \Omega} \sum_{P} \frac{\theta\left(\omega_{D}-\left|\xi_{\vec{p}}\right|\right)}{\left(i \nu_{n}-\xi_{\vec{p}_{+}}\right)\left(-i \nu_{n}-\xi_{\vec{p}_{-}}\right)-E}
$$

where $\vec{p}_{ \pm}=\frac{\vec{k}}{2} \pm \vec{p}$. For the pairing mode, we expand the r. h. s. to the first order in $E$ and to the second order in $\vec{k}$. After some manipulations, we end up with

$$
E \simeq \frac{8 \pi^{2}\left(k_{B} T_{C}\right)^{2}}{7 \zeta(3)} \frac{T-T_{C}}{T_{C}}+\frac{1}{6} v_{F}^{2} \vec{k}^{2}
$$

On writing $\Psi_{\vec{k}} u_{\vec{k}}(P)$ for the order parameter at a nozero $\vec{k}$ with $u_{\vec{k}}(P)$ being the pairing mode at $\vec{k} \neq 0$, the free energy functional becomes

$$
\Gamma(\Psi)=\Omega\left[\left[\frac{1}{6} v_{F}^{2} \vec{k}^{2}+\frac{8 \pi^{2}\left(k_{B} T_{C}\right)^{2}}{7 \zeta(3)} \frac{T-T_{C}}{T_{C}}\right]\left|\Psi_{\vec{k}}\right|^{2}+\frac{8 \pi^{4} v_{F}\left(k_{B} T_{C}\right)^{2}}{7 \zeta(3) k_{F}^{2}}\left|\Psi_{\vec{k}}\right|^{4}\right]
$$


By summing over $\vec{k}$ and introducing

$$
\Phi(\vec{r})=\sqrt{\frac{2 \mu}{3}} \int \frac{d^{3} \vec{k}}{(2 \pi)^{3}} \Psi_{\vec{k}} e^{i \vec{k} \cdot \vec{r}}
$$

we obtain, for an inhomogeneous order parameter, that

$$
\Gamma=\int d^{3} \vec{r}\left[\frac{1}{2 m} \vec{\nabla} \Phi^{*} \vec{\nabla} \Phi+a|\Phi|^{2}+\frac{1}{2} b|\Phi|^{4}\right]
$$

with coefficients

$$
a=\frac{12 \pi^{2}}{7 \zeta(3) \mu} k_{B}^{2} T_{C}\left(T-T_{C}\right)
$$

and

$$
b=\frac{36 \pi^{4} v_{F}}{7 \zeta(3)} \frac{\left(k_{B} T_{C}\right)^{2}}{\mu^{2} k_{F}^{2}},
$$

which is in agreement with the result obtained by Gorkov [13].

\section{Appendix B.}

In order to determine the vertex contribution to the coefficient $c^{\prime}\left(T_{c}, \mu\right)$ in eq. (6.6), we approximate $\bar{\Gamma}_{A, \vec{k}}\left(P \mid P^{\prime}\right)$ by considering only the one magnetic gluon exchange, with both incoming and outgoing quark momenta near the Fermi surface, i. e.

$$
\bar{\Gamma}_{A, \vec{k}}\left(P \mid P^{\prime}\right) \simeq-\frac{i}{g^{2}}\left(1+\frac{1}{N}\right) D_{M}(\vec{q}, \omega) \bar{u}\left(\vec{p}_{+}^{\prime}\right) \gamma_{i} u\left(\vec{p}_{+}\right) \bar{u}\left(\vec{p}_{-}^{\prime}\right) \gamma_{j} u\left(\vec{p}_{-}\right)\left(\delta_{i j}-\hat{q}_{i} \hat{q}_{j}\right)
$$

where $u(\vec{p})$ is a solution of the Dirac equation $\left(\gamma_{4} p-i \vec{\gamma} \cdot \vec{p}\right) u(\vec{p})=0 u^{\dagger}(\vec{p}) u(\vec{p})=1$, and

$$
\vec{p}_{ \pm}=\frac{\vec{k}}{2} \pm \vec{p}, \quad \vec{p}_{ \pm}^{\prime}=\frac{\vec{k}}{2} \pm \vec{p}^{\prime}, \quad \vec{q}=\vec{p}-\vec{p}^{\prime}
$$

with $p \sim p^{\prime} \sim \mu$, and $D_{M}(\vec{q}, \omega)$ contains the HDL resummation. With the aid of the Dirac equation, (10.1) can be reduced to

$$
\bar{\Gamma}_{A, \vec{k}}\left(P \mid P^{\prime}\right) \simeq-\frac{i}{g^{2}}\left(1+\frac{1}{N}\right) D_{M}(\vec{q}, \omega)\left[V-\frac{\left(\vec{p}_{+}^{\prime}-\vec{p}_{+}\right)\left(\vec{p}_{-}^{\prime}-\vec{p}_{-}\right)}{\vec{q}^{2}} S\right]
$$

where

$$
\begin{aligned}
& V=-u^{\dagger}\left(\vec{p}_{+}^{\prime}\right) \vec{\alpha} u\left(\vec{p}_{+}\right) u^{\dagger}\left(\vec{p}_{-}^{\prime}\right) \vec{\alpha} u\left(\vec{p}_{-}\right) \\
& S=u^{\dagger}\left(\vec{p}_{+}^{\prime}\right) u\left(\vec{p}_{+}\right) u^{\dagger}\left(\vec{p}_{-}^{\prime}\right) u\left(\vec{p}_{-}\right)
\end{aligned}
$$


with $\vec{\alpha}=\left(\begin{array}{cc}0 & \vec{\tau} \\ \vec{\tau} & 0\end{array}\right)$ and $\vec{\tau}$ the $2 \times 2$ Pauli matrices. Since $u(\vec{p})$ is also an eigenstate of $\gamma_{5}=\left(\begin{array}{cc}0 & -I \\ -I & 0\end{array}\right)$ we have

$$
u(\vec{p})=\frac{1}{\sqrt{2}}\left(\begin{array}{c}
\phi(\vec{p}) \\
\pm \phi(\vec{p})
\end{array}\right)
$$

with $\vec{\tau} \hat{p} \phi(\vec{p})=\phi(\vec{p})$. Using the identity $\left(\tau^{j}\right)_{\alpha \beta}\left(\tau^{j}\right)_{\rho \lambda}=2 \delta_{\alpha \lambda} \delta_{\beta \rho}-\delta_{\alpha \beta} \delta_{\rho \lambda}$ we find that

$$
V=S-2 S^{\prime}, \quad S^{\prime}=\phi^{\dagger}\left(\vec{p}_{+}^{\prime}\right) \phi\left(\vec{p}_{-}\right) \phi^{\dagger}\left(\vec{p}_{-}^{\prime}\right) \phi\left(\vec{p}_{+}\right)
$$

In the collinear limit, $\vec{q} \mapsto 0, \vec{p}_{ \pm} \mapsto \vec{p}_{ \pm}^{\prime}$, we have $S \mapsto 1$ and

$$
S^{\prime} \mapsto \frac{1}{2}\left(1+\hat{p}_{+} \hat{p}_{-}\right) \simeq \frac{k^{2}}{p^{2}}\left[1-(\hat{p} \hat{k})^{2}\right]
$$

Therefore, a net momentum $\vec{k}$ shifts the kernel $K\left(P \mid P^{\prime}\right)$ by an amount

$$
\delta^{\prime \prime} K\left(P \mid P^{\prime}\right) \sim-K\left(P \mid P^{\prime}\right) \frac{k^{2}}{4 p^{2}}\left[1-\left(\hat{p}^{\prime} \hat{k}\right)^{2}\right]
$$

Comparing $\delta^{\prime \prime} K\left(P \mid P^{\prime}\right)$ with the second term of $\delta^{\prime} K\left(P \mid P^{\prime}\right)$ in (6.5), we notice that although the former differs by a factor of $\nu_{n}{ }^{2}+(p-\mu)^{2}$ in the denominator in comparison with the latter, it compensates by the factor of $p^{2} \sim \mu^{2}$ which appears in the denominator. As the sum over Matsubara energies will pick up the infrared logarithm, $\ln \frac{1}{\epsilon}$, we expect that the contributuion of $\delta^{\prime \prime} K\left(P \mid P^{\prime}\right)$ to $c^{\prime}\left(T_{c}, \mu\right)$ is suppressed relative to that of of $\delta^{\prime} K\left(P \mid P^{\prime}\right)$ by a factor of order

$$
\left(\frac{k_{B} T_{c}}{\mu}\right)^{2} \ln \frac{\mu}{k_{B} T_{c}} \ll 1
$$

and therefore can be ignored.

\section{Appendix C.}

By applying the variational principle to the free energy functional we obtain the equation of motion for the special case of $\Phi_{l}=\vec{A}_{l}=0$ for $l \neq 0,8$ and $\overrightarrow{\mathcal{V}}=0$,

$$
\begin{aligned}
& \vec{\nabla} \times(\vec{\nabla} \times \vec{V})=\vec{J} \\
& -(\vec{\nabla}-2 i \lambda \vec{V})^{2} \phi+4 a \phi+2 b\left(2|\phi|^{2}+|\chi|^{2}\right) \phi=0 \\
& -(\vec{\nabla}+i \lambda \vec{V})^{2} \chi+4 a \chi+2 b\left(|\phi|^{2}+\frac{3}{2}|\chi|^{2}\right) \chi=0
\end{aligned}
$$


with the current vector given by

$$
\vec{J}=i \lambda\left[-2 \phi^{\star}(\vec{\nabla}-2 i \lambda \vec{V}) \phi+\chi^{\star}(\vec{\nabla}+i \lambda \vec{V}) \chi\right]+\cdots
$$

and the $\cdots$ indicate the complex conjugate. For a vortex filament parallel to $\hat{\zeta}$, we make the following ansatz for the solution in cylindrical coordinates

$$
\phi=u(\rho) e^{2 n i \theta}, \quad \chi=v(\rho) e^{-n i \theta}, \quad \vec{V}=V(\rho) \hat{\theta}
$$

The set of equations (11.1) is reduced to a set of ordinary non-linear differential equations

$$
\begin{aligned}
& -\frac{d}{d \rho}\left[\frac{1}{\rho} \frac{d}{d \rho}(\rho V)\right]=2 \lambda\left(\frac{n}{\rho}-\lambda V\right)\left(4 u^{2}+v^{2}\right) \\
& -\frac{d^{2} u}{d \rho^{2}}-\frac{1}{\rho} \frac{d u}{d \rho}+4\left(\frac{n}{\rho}-\lambda V\right)^{2} u+4 a u+2 b\left(2 u^{2}+v^{2}\right) u=0 \\
& -\frac{d^{2} v}{d \rho^{2}}-\frac{1}{\rho} \frac{d v}{d \rho}+\left(\frac{n}{\rho}-\lambda V\right)^{2} v+4 a v+2 b\left(u^{2}+\frac{3}{2} v^{2}\right) v=0
\end{aligned}
$$

If the color and the flavor were locked everywhere, we would have $v=\sqrt{2} u$. The last two equations would then imply that

$$
V=\frac{n}{\lambda \rho}
$$

which is a pure gauge for $\rho \neq 0$, as the total flux has been fixed by the phase of $\phi$ and $\chi$ in (11.3). A complete color-flavor locking would squeeze all flux in the symmetry axis of the filament and would make the magnetic field energy per length infinite.

The next example is the equilibrium between the super phase and the normal phase under an external $V$-field, the classical problem of the surface tension of the interface which was worked out by Ginzburg and Landau for electronic superconductors [24]. With the interface parallel to the $y-\zeta$ plane, the solution ansatz reads $\phi=u(x), \chi=v(x)$ and $\vec{V}=V(x) \hat{\zeta}$, where $u, v$ are real and positive. The set of equations (11.1) reduces to

$$
\begin{gathered}
\frac{d^{2} V}{d x^{2}}=2 \lambda^{2} V\left(4 u^{2}+v^{2}\right) \\
-\frac{d^{2} u}{d x^{2}}+4 \lambda^{2} V^{2} u+4 a u+2 b\left(2 u^{2}+v^{2}\right) u=0 \\
-\frac{d^{2} v}{d x^{2}}+\lambda^{2} V^{2} v+4 a v+2 b\left(u^{2}+\frac{3}{2} v^{2}\right) v=0
\end{gathered}
$$

An inspection of the last two equations shows that a complete color-flavor locking, $v=\sqrt{2} u$ can not implemented unless $V=0$. 


\section{References.}

[1] B. Barrois, Nucl. Phys. B129(1977) 390, S. Frautschi, Proceedings of the Workshop on Hadronic Matter at Extreme Energy Density, N. Cabibbo, Editor, Erice, Italy, 1978, D. Bailin and A. Love, Phys. Rep. 107 (1984) 325, and references therein for early works.

[2] T. Schafer and E. V. Shuryak, Rev. Mod. Phys. 70 (1998) 323.

[3] M. Alford, K. Rajagopal and F. Wilczek, Nucl. Phys. B537 (1999) 443, M. Alford, hep-th/0102047, K. Rajagopal and Wilczek, hep-ph/0011333, to appear in B. L. Ioffe Festschrift, "At the Frontier of Particle Physics/Handbook of QCD" M. Shifman ed., (World Scientific 2001), and the references therein

[4] T. Schafer and F. Wilczek, Phys. Rev. Lett. 82 (1999) 3956.

[5] D. T. Son, Phys. Rev. D59 (1999) 094019.

[6] T. Schafer and F. Wilczek, Phys. Rev. D60 (1999) 114033.

[7] R. D. Pisarski and D. H. Rischke, Phys. Rev. D61 (2000) 051501.

[8] R. D. Pisarski and D. H. Rischke, Phys. Rev. D61 (2000) 074017.

[9] D. K. Hong, Nucl. Phys. B582 (2000) 451.

[10] W. Brown, J. T. Liu, H. C. Ren, Phys. Rev. D61 (2000) 114012.

[11] W. Brown, J. T. Liu and H. C. Ren, Phys. Rev. D62 (2000) 054016, Phys. Rev. D62 (2000) 054013.

[12] S. D. Hsu and M. Schwetz Nucl. Phys. B572 (2000) 211.

[13] L. P. Gorkov, Soviet Physics JETP 36(9), No. 6, (1959) 1364.

[14] G. Jona-Lasinio, Nuovo Cimento B26 (1975) 99.

[15] K. Iida and G. Baym, Phys. Rev. D63 (2001) 074018.

[16] T. Schafer, Nucl. Phys. B575 (2000) 269.

[17] I. A. Shovkovy and L. C. R. Wijewardhana, Phys. Lett. $470 B$ (1999), 189.

[18] D. H. Rischke and R. D. Pisarski, Color Superconductivity in Cold, Dense Quark Matter, Proceedings of the 5th Workshop on QCD, Villefrance-sur-Mer, France, 3-7 January 2000.

[19] M. Alford, K. Rajagopal and F. Wilczek, Phys. Lett. 422B (1998) 247.

[20] R. Rapp, T. Schafer, E. Shuryak and M. Velkovsky, Phys. Rev. Lett. 81 (1998) 53.

[21] M. Alford, J. Berges and K. Rajagopal, Nucl. Phys. B571 (2000) 269.

[22] K. Rajagopal and F. Wilczek Phys. Rev. Lett. 86 (2001) 3492.

[23] Y. Ren, J. H. Xu and C. S. Ting, Phys. Rev. Lett. 74 (1995), 3680.

[24] V. L. Ginzburg and L. D. Landau, Zh. Eksp. Teor. Fiz 20 (1950) 1064. 


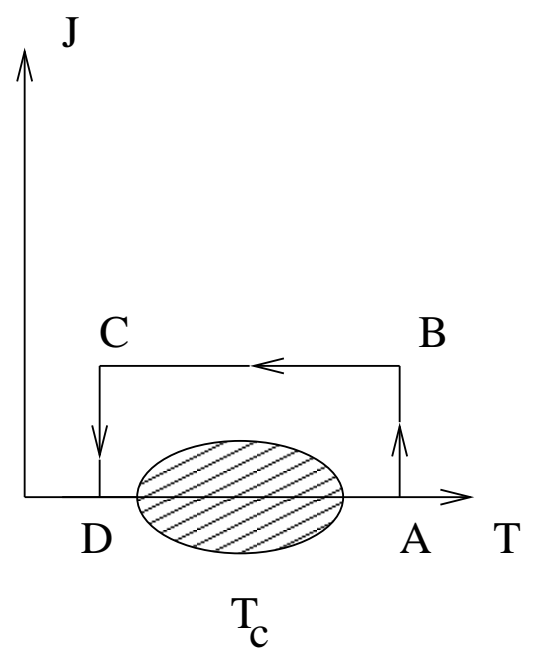

(a)

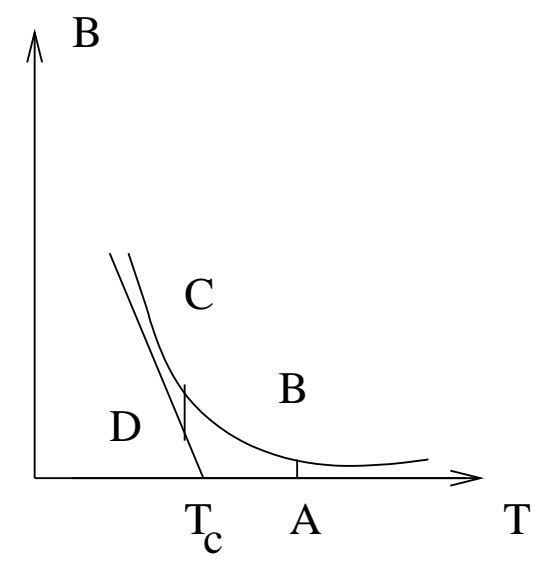

(b)

Figure 1

The continuation from the normal phase to the super phase

a) The continuation path on $T-J$ plane

b) The order parameter versus temperature

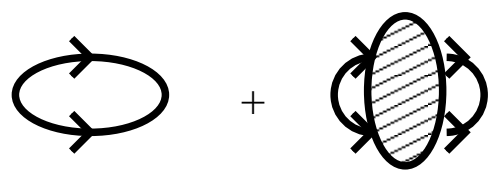

(a)

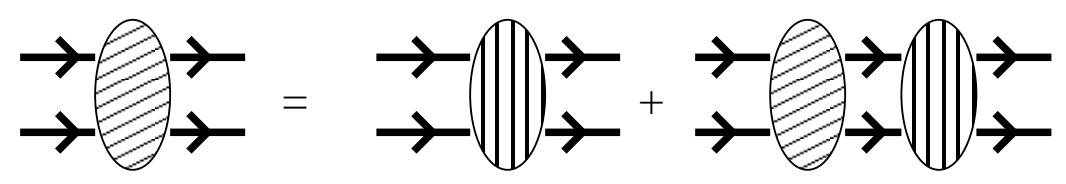

(b)

Figure 2

a) Diagram of a quark pair propagator. The thick black line indicates the full quark pair propagator. 
b) Dyson-Schwinger equation for the proper vertex of diquark scattering, where the vertically shaded vertex stands for two quark irreducible diagrams.

$1 / 2$
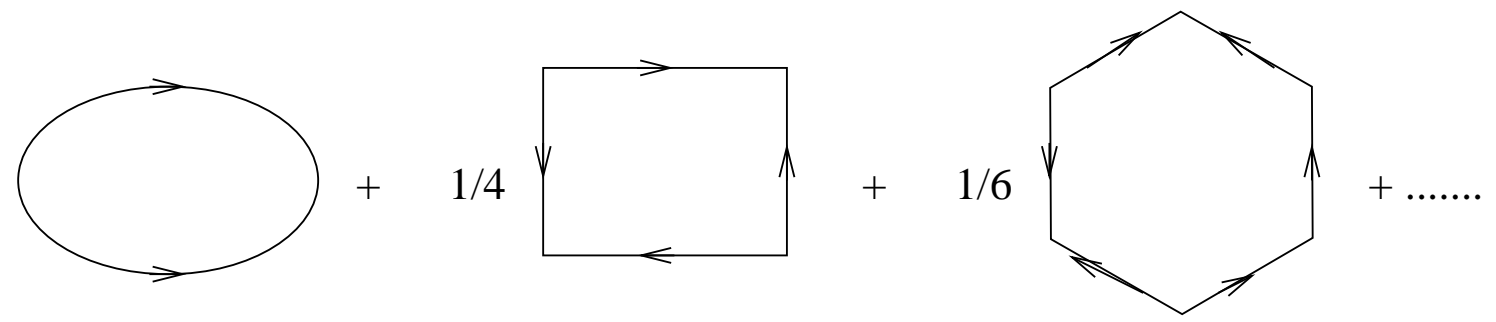

Figure 3

The diagrammatic expansion of $\mathrm{W}(\mathrm{J})$ at $\mathrm{g}=0$. 